\title{
A Chiral Ag-based Catalyst for Practical, Efficient and Highly Enantioselective Additions of Enolsilanes to $\alpha$-Ketoesters
}

\author{
Laura C. Akullian, Marc L. Snapper* and Amir H. Hoveyda* \\ Department of Chemistry, Merkert Chemistry Center, Boston College, Chestnut Hill, Massachusetts 02467
}

\section{SUPPORTING INFORMATION}

General. Infrared (IR) spectra are recorded on a Perkin Elmer 781 spectrophotometer, $v_{\max }$ in $\mathrm{cm}^{-1}$. Bands are characterized as broad (br), strong (s), medium (m) or weak (w). ${ }^{1} \mathrm{H}$ NMR spectra are recorded on a Varian Unity INOVA $400(400 \mathrm{MHz})$ spectrometer. Chemical shifts are reported in ppm from the solvent resonance resulting from incomplete deuteration as the internal standard $\left(\mathrm{CDCl}_{3}: \delta 7.26\right)$. Data are reported as follows: chemical shift, integration, multiplicity $(\mathrm{s}=$ singlet, $\mathrm{d}=$ doublet, $\mathrm{t}=$ triplet, $\mathrm{q}=$ quartet, $\mathrm{br}=$ broad, $\mathrm{m}=$ multiplet $)$, and coupling constants. ${ }^{13} \mathrm{C}$ NMR spectra are recorded on a Varian Unity INOVA 400 (100 MHz) with complete proton decoupling. Chemical shifts are reported in ppm with the solvent resonance as the internal standard ( $\left.\mathrm{CDCl}_{3}: \delta 77.16\right)$. Enantiomer ratios are determined by chiral GLC analysis; (Alltech Associates Chiraldex GTA column (30 m x 0.25 mm) or Supelco Betadex 120 column (30 m x $0.25 \mathrm{~mm}$ )), or by chiral HPLC analysis (Chiral Technologies Chiralpak OD column $(0.46 \mathrm{~cm} \times 25 \mathrm{~cm})$ and AS $(0.46 \mathrm{~cm} \times 0.25 \mathrm{~cm})$ in comparison with authentic racemic materials. High-resolution mass spectrometry was performed on a Micromass LCT ESI-MS (positive mode) at the Mass Spectrometry Facility at Boston College or at the University of Illinois at Urbana-Champaign. Elemental analyses were carried out at Roberson Microlit Laboratories, Inc. Madison, NJ. Optical rotation values were recorded on a Rudolph Research Analytical Autopol IV polarimeter.

Unless otherwise stated, all reactions were conducted in oven- $\left(135{ }^{\circ} \mathrm{C}\right)$ and flame-dried glassware under an inert atmosphere of dry $\mathrm{N}_{2}$. Solvents were purified under a positive pressure of dry Ar by a modified Advanced ChemTech purification system; dichloromethane was purified through two alumina columns. Tetrahydrofuran was distilled from Na and benzophenone, unless otherwise stated. $\mathrm{AgF}$ and $\mathrm{AgF}_{2}$ were purchased from Aldrich and used without purification. Enolsilanes were purchased from commercial sources (Fluka, Aldrich) and used without purification. $\alpha$-Ketoesters were purchased from commercial sources (Aldrich, Lancaster), or synthesized according to previous procedures, ${ }^{1}$ and purified by simple distillation. $\mathrm{EDC} \cdot \mathrm{HCl}$, $\mathrm{HOBt} \cdot \mathrm{H}_{2} \mathrm{O}$, 4.0 M solution of $\mathrm{HCl}$ in 1,4-dioxane, $n$-butylamine, Boc-protected amino acids, and pyridine carboxaldehydes were purchased from commercial sources (Advanced Chemtech, Nova Biochem) and used without purification. Unless otherwise stated, substrates were synthesized from commercially available starting materials through previously reported protocols.

1) Rambaud, M.; Bakasse, M.; Duguay, G.; Villieras, J. Synthesis 1988, 7, 564-566. 
(S)-N-((S)1-(butylamino)1-oxo-3-phenylpropan-2-yl)3,3-dimethyl-2-((E)(6-methylpyridin-2yl)methyleneamino)butanamide (5d). Boc-L-Phe-OH (3.00 g, $11.31 \mathrm{mmol})$ is dissolved in $50 \mathrm{~mL}$ of $\mathrm{CH}_{2} \mathrm{Cl}_{2}$, and $\mathrm{HOBt} \cdot \mathrm{H}_{2} \mathrm{O}(1.90 \mathrm{~g}, 12.43 \mathrm{mmol}), \mathrm{EDC} \cdot \mathrm{HCl}(2.38 \mathrm{~g}, 12.43 \mathrm{mmol})$ and butylamine $(2.75 \mathrm{~mL}, 27.72 \mathrm{mmol})$ are added successively at $22{ }^{\circ} \mathrm{C}$. The resulting solution is allowed to stir at $22{ }^{\circ} \mathrm{C}$ for $4 \mathrm{~h}$. Citric acid (50 mL of a $10 \mathrm{wt}$ \% aqueous solution) is added and the two layers are separated. The aqueous layer is washed twice with $50 \mathrm{~mL}$ of $\mathrm{CH}_{2} \mathrm{Cl}_{2}$ and the combined organic layers are washed with $50 \mathrm{~mL} 10 \mathrm{wt}$. \% aqueous citric acid and $50 \mathrm{~mL}$ of saturated aqueous $\mathrm{NaHCO}_{3}$, followed by $50 \mathrm{~mL}$ of a saturated solution of $\mathrm{NaCl}$. The organic layer is dried over $\mathrm{MgSO}_{4}$ before removal of the volatiles in vacuo to afford Boc-L-Phe-NHBu as a white solid (3.58 g, $11.19 \mathrm{mmol}$, 90\% yield). Hydrochloric acid (4.0 $\mathrm{M}$ solution in 1,4-dioxane) is added dropwise to Boc-L-Phe-NHBu $(2.0 \mathrm{~g}, 6.24 \mathrm{mmol})$ at $0{ }^{\circ} \mathrm{C}$ until complete dissolution is achieved. The resulting solution is allowed to warm to $22{ }^{\circ} \mathrm{C}$ and stir for $30 \mathrm{~min}$. At this time, the mixture is concentrated in vacuo to afford $\mathrm{ClH}_{3} \mathrm{~N}-\mathrm{L}-\mathrm{Phe}-\mathrm{NHBu}(1.41 \mathrm{~g}, 5.49$ mmol, 88\% yield).

Boc-L-Tle-OH (0.501 g, $1.26 \mathrm{mmol})$ is dissolved in $10 \mathrm{~mL} \mathrm{CH}_{2} \mathrm{Cl}_{2}$ and $\mathrm{HOBt} \bullet \mathrm{H}_{2} \mathrm{O}(0.212 \mathrm{~g}$, $1.39 \mathrm{mmol}), \mathrm{EDC} \cdot \mathrm{HCl}(0.266 \mathrm{~g}, 1.39 \mathrm{mmol}), \mathrm{ClH}_{3} \mathrm{~N}-\mathrm{L}-\mathrm{Phe}-\mathrm{NHBu}(0.324 \mathrm{~g}, 1.26 \mathrm{mmol})$, and $\mathrm{Et}_{3} \mathrm{~N}$ (3.86 mL, $2.77 \mathrm{mmol}$ ) are successively added at $22{ }^{\circ} \mathrm{C}$. The resulting solution is allowed to stir at $22^{\circ} \mathrm{C}$ for $4 \mathrm{~h}$ before quenching and work-up as described above. Boc-L-Tle-L-Phe-NHBu is dissolved in $4.0 \mathrm{M} \mathrm{HCl}$ in 1,4 -dioxane at $0{ }^{\circ} \mathrm{C}$. The solution is allowed to warm to $22{ }^{\circ} \mathrm{C}$ and stirred for $30 \mathrm{~min}$. The mixture is concentrated in vacuo and the resulting solid dissolved in water. $\mathrm{NaOH}$ (1 $\mathrm{N}$ solution) is added dropwise until $\mathrm{pH}>10$ is achieved, after which the aqueous solution is washed with $\mathrm{CH}_{2} \mathrm{Cl}_{2}(3 \times 20 \mathrm{~mL})$. The combined organic layers are dried over $\mathrm{MgSO}_{4}$ and concentrated to afford $\mathrm{H}_{2} \mathrm{~N}$-L-Tle-L-Phe-NHBu as a light tan solid $(0.357 \mathrm{~g}$, $1.07 \mathrm{mmol}, 85 \%$ yield). The purity of $\mathrm{H}_{2} \mathrm{~N}-\mathrm{L}-\mathrm{Tle}-\mathrm{L}-\mathrm{Phe}-\mathrm{NHBu}$ is established by inspection of its $400 \mathrm{MHz}{ }^{1} \mathrm{H}$ NMR spectrum before the final coupling.

6-Methyl-2-pyridine carboxaldehyde $(0.130 \mathrm{~g}, 1.07 \mathrm{mmol})$ is dissolved in $5 \mathrm{~mL}$ of $\mathrm{CH}_{2} \mathrm{Cl}_{2}$. $\mathrm{H}_{2} \mathrm{~N}$-L-Tle-L-Phe-NHBu (0.357 g, $\left.1.07 \mathrm{mmol}\right)$ dissolved in $5 \mathrm{~mL} \mathrm{CH}_{2} \mathrm{Cl}_{2}$ and $\mathrm{MgSO}_{4}$ are added at $22{ }^{\circ} \mathrm{C}$. The resulting solution is allowed to stir for $3 \mathrm{~h}$, after which $\mathrm{MgSO}_{4}$ is removed by filtration and the organic filtrate is concentrated in vacuo. The resulting residue is purified by recrystallization (pentane/ $\left.\mathrm{Et}_{2} \mathrm{O}\right)$ to afford $\mathbf{5 d}$ as white crystalline solid $(0.252 \mathrm{~g}, 0.578 \mathrm{mmol}$, 54\% yield). m.p. = $88.2^{\circ} \mathrm{C}$; IR (solid film): 3298 (m), 2958 (m), 2932 (w), 2870 (w), 1646 (s), 1577 (w), 1509 (m), 1457 (w), 1366 (w) cm ${ }^{-1}$; ${ }^{1} \mathrm{NMR}$ (400 MHz): $\delta 8.16(1 \mathrm{H}, \mathrm{s}), 7.78(1 \mathrm{H}, \mathrm{d}$, $J=7.6 \mathrm{~Hz}), 7.67(1 \mathrm{H}, \mathrm{t}, J=7.6 \mathrm{~Hz}), 7.33-7.21(\mathrm{~m}, 6 \mathrm{H}), 7.17(1 \mathrm{H}$, br d, $J=7.6 \mathrm{~Hz}), 5.79(1 \mathrm{H}$, br s), $4.65(1 \mathrm{H}, \mathrm{q}, J=7.6 \mathrm{~Hz}), 3.54(1 \mathrm{H}, \mathrm{s}), 3.25-3.20(1 \mathrm{H}, \mathrm{m}), 3.14-3.02(3 \mathrm{H}, \mathrm{m}), 2.59(3 \mathrm{H}, \mathrm{s})$, 1.28-1.19 (2H, m), 1.14-1.05 (2H, m), $0.89(9 \mathrm{H}, \mathrm{s}), 0.76(3 \mathrm{H}, \mathrm{t}, J=7.2 \mathrm{~Hz})$; ${ }^{13} \mathrm{C} \mathrm{NMR}$ (100 MHz): $\delta$ 171.6, 170.6, 163.5, 158.4, 153.3, 137.1, 137.0, 129.5, 128.9, 127.1, 125.0, 118.3, 83.1, 54.3, 39.3, 38.1, 35.5, 31.4, 27.2, 24.4, 20.0, 13.7; HRMS calcd for $\mathrm{C}_{26} \mathrm{H}_{37} \mathrm{~N}_{4} \mathrm{O}_{2}\left(\mathrm{M}+\mathrm{H}^{+}\right)$: 437.2925, Found: 437.2917; $[\alpha]_{\mathrm{D}}{ }^{20}=-10.31\left(c=0.79, \mathrm{CHCl}_{3}\right)$.

Representative experimental procedure for enantioselective Ag-catalyzed additions of enolsilanes to $\alpha$-ketoesters: A 16 x $100 \mathrm{~mm}$ test tube, equipped with a magnetic stir bar, is 
charged with $\mathrm{AgF}_{2}(1.8 \mathrm{mg}, 0.012 \mathrm{mmol})$ and then chiral ligand 5d (5.2 $\mathrm{mg}, 0.012 \mathrm{mmol}$ ) followed by tetrahydrofuran $(0.40 \mathrm{~mL})$. The resulting solution is allowed to stir while ethyl-2oxo-4-phenylbutyrate (29 $\mu \mathrm{L}, 0.12 \mathrm{mmol}$ ) is added, followed by the addition of $0.40 \mathrm{~mL}$ of tetrahydrofuran. The test tube is sealed with a septum, purged with $\mathrm{N}_{2}$ and allowed to stir for 20 min. At this time, the solution is allowed to cool to $-78{ }^{\circ} \mathrm{C}$ in a dry ice/acetone bath and a solution of 1-phenyl-1-trimethylsiloxyethylene (29 $\mu \mathrm{L}, 0.14 \mathrm{mmol}$ ) dissolved in $0.40 \mathrm{~mL}$ tetrahydrofuran is added dropwise through syringe. The test tube is sealed with teflon tape and the mixture is allowed to warm to $-30{ }^{\circ} \mathrm{C}$ in a circulating bath. The mixture is allowed to stir ${ }^{2}$ for $24 \mathrm{~h}$ at $-30{ }^{\circ} \mathrm{C}$, after which $1 \mathrm{~mL}$ of a $2.0 \mathrm{M}$ solution of $\mathrm{HCl}$ is added and the mixture is allowed warm to $22^{\circ} \mathrm{C}$. The mixture is allowed to stir for $10 \mathrm{~min}$ at $22^{\circ} \mathrm{C}$. The aqueous layer is washed with $\mathrm{Et}_{2} \mathrm{O}(3 \times 1.0 \mathrm{~mL}$ ) and the combined organic layers are filtered through silica gel and volatiles are removed under reduced pressure to afford pale yellow oil. The oil is purified by silica gel chromatography (5:1 pentane: $\mathrm{Et}_{2} \mathrm{O}$ ) to give the desired aldol addition product (Table 2, entry 1) as a colorless oil (36 mg, $0.11 \mathrm{mmol}$, 92\% yield).

Ethyl-(R)-2-hydroxy-4-oxo-2-phenethyl-4-phenylbutanoate (Table 2, entry 1). The mixture is allowed to stir at $-40{ }^{\circ} \mathrm{C}$ for $48 \mathrm{~h}$. Following purification, the desired product is obtained as colorless oil. IR (neat): 3515 (br), 2962 (s), 2923 (w), 1728 (s), 1671 (s), 1451 (w), 1260 (s), 1216 (s), 1090 (s), 1022 (s), 799 (s) cm ${ }^{-1}$; ${ }^{1} \mathrm{H}$ NMR (400 MHz): $\delta$ 7.94-7.92 (2H, m), 7.59 (1H, tt, $J=7.2,1.6 \mathrm{~Hz}), 7.49-7.45(2 \mathrm{H}, \mathrm{m}), 7.32-7.28$ (2H, m), 7.22-7.19 (3H, m), 4.22 (2H, dq, $J=7.2$, $0.4 \mathrm{~Hz}), 3.99(1 \mathrm{H}, \mathrm{br}$ s), $3.63(1 \mathrm{H}, \mathrm{d}, J=17.6 \mathrm{~Hz}), 3.49(1 \mathrm{H}, \mathrm{d}, J=17.6), 2.93-2.86(1 \mathrm{H}, \mathrm{m})$, 2.61-2.53 (1H, m), 2.12-2.08 (2H, m), $1.28(3 \mathrm{H}, \mathrm{t}, J=7.2 \mathrm{~Hz}) .{ }^{13} \mathrm{C}$ NMR $(100 \mathrm{MHz}): \delta 198.6$, 175.4, 141.6, 136.7, 133.8, 128.8, 128.6, 128.5, 128.3, 126.2, 75.1, 62.0, 47.4, 41.5, 29.6, 14.3; HRMS calcd for $\mathrm{C}_{20} \mathrm{H}_{22} \mathrm{O}_{4} \mathrm{Na}\left(\mathrm{M}+\mathrm{Na}^{+}\right)$: 349.1416, Found: 349.1436; Anal Calcd for $\mathrm{C}_{20} \mathrm{H}_{22} \mathrm{O}_{4}$ : C, 73.60; H, 6.79; Found: C, 73.50; H, 6.36; $[\alpha]_{\mathrm{D}}{ }^{20}=-26.50\left(c=1.00, \mathrm{CHCl}_{3}\right)$.

Optical purity of the aldol adduct was established by chiral HPLC analysis: Chiracel OD (0.46 $\mathrm{x}$ $25 \mathrm{~cm})$, 96:4 hexanes:i-PrOH, $1.0 \mathrm{~mL} / \mathrm{min}, \lambda=254 \mathrm{~nm}, 86 \%$ ee.
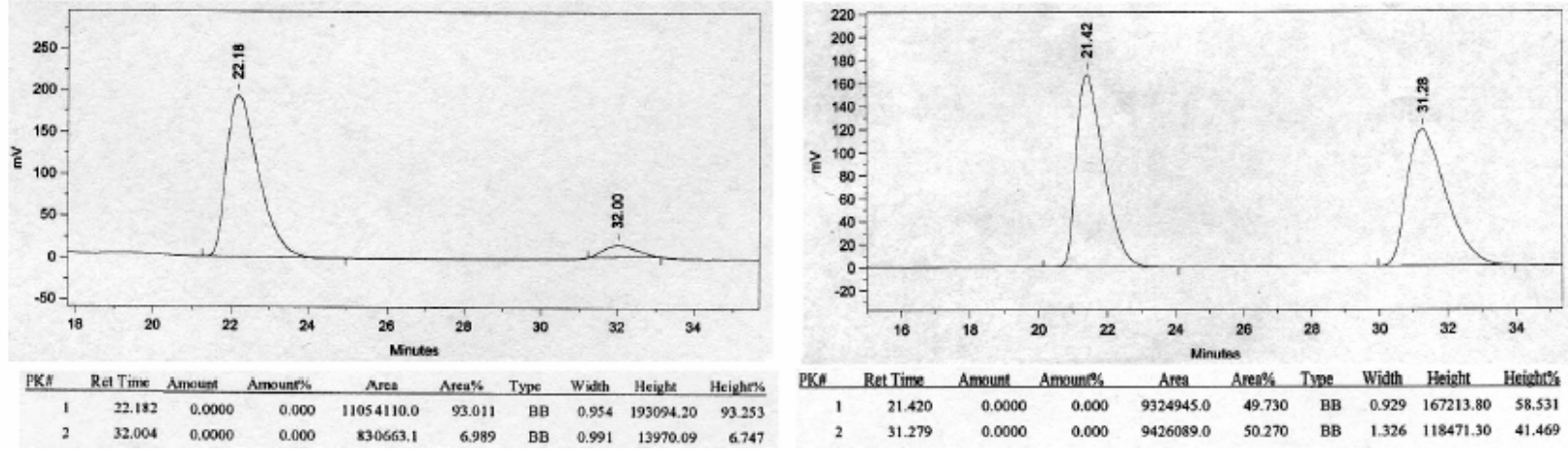

Dimethyl-(R)-2-hydroxy-2-(2-oxo-2-phenylethyl)pentanedioate (Table 2, entry 2). The mixture was allowed to stir at $-30{ }^{\circ} \mathrm{C}$ for $24 \mathrm{~h}$. Addition of water followed by washing with $\mathrm{Et}_{2} \mathrm{O}$ and concentration at reduced pressure affords the desired adduct. The purified product was

2) The heterogeneous mixture requires constant stirring so that $>98 \%$ conversion can be achieved. 
obtained as a white solid. m.p. = $70.5^{\circ} \mathrm{C}$; IR (neat): 3496 (br), 2955 (s), 1734 (s), 1685 (s), 1449 (m), 1218 (s) cm ${ }^{-1} ;{ }^{1} \mathrm{H}$ NMR (400 MHz): $\delta$ 7.93-7.91 (2H, m), $7.59(1 \mathrm{H}, \mathrm{tt}, J=7.2,1.2 \mathrm{~Hz})$, 7.49-7.45 (2H, m), 3.90 (1H, br s), 3.71 (3H, s), 3.68 (3H, s), 3.61 (1H, d, $J=17.6 \mathrm{~Hz}), 3.46$ $(1 \mathrm{H}, \mathrm{d}, J=17.6 \mathrm{~Hz}), 2.63-2.55(1 \mathrm{H}, \mathrm{m}), 2.38-2.30(1 \mathrm{H}, \mathrm{m}), 2.16-2.12(2 \mathrm{H}, \mathrm{m}) ;{ }^{13} \mathrm{C}$ NMR $(100$ MHz): $\delta$ 198.5, 175.6, 173.7, 136.5, 134.0, 128.9, 128.4, 74.5, 53.1, 52.0, 47.5, 34.3, 28.3; HRMS calcd for $\mathrm{C}_{15} \mathrm{H}_{18} \mathrm{O}_{6} \mathrm{Na}\left(\mathrm{M}+\mathrm{Na}^{+}\right)$: 317.0991, Found: 317.1001; $[\alpha]_{\mathrm{D}}{ }^{20}=-24.42(c=1.49$, $\left.\mathrm{CHCl}_{3}\right)$.

Optical purity of the product was established by chiral HPLC analysis: Chiracel AS (0.46 x 25 cm), 95:5 hexanes:i-PrOH, $1.0 \mathrm{~mL} / \mathrm{min}, \lambda=254 \mathrm{~nm}$; 92\% ee.
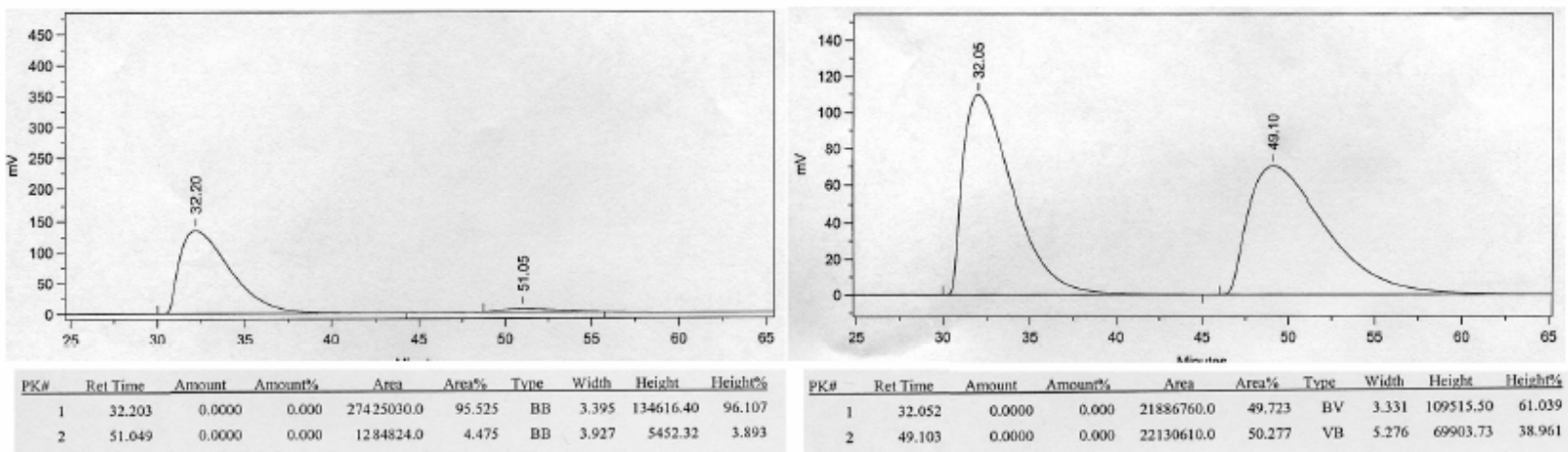

Ethyl-(R)-2-hydroxy-4-methyl-2-(2-oxo-2-phenylethyl)pentanoate (Table 2, entry 3). The mixture is allowed to stir at $-30{ }^{\circ} \mathrm{C}$ for $24 \mathrm{~h}$. Following purification, the desired product is obtained as colorless oil. IR (neat): 3515 (br), 2956 (s), 1736 (s), 1686 (s), 1596 (w), 1449 (m), $1363(\mathrm{w}), 1219$ (s) $\mathrm{cm}^{-1}$; ${ }^{1} \mathrm{H}$ NMR (400 MHz): $\delta$ 7.94-7.91 (2H, m), $7.58(1 \mathrm{H}, \mathrm{tt}, J=7.2,1.2 \mathrm{~Hz})$, 7.49-7.44 (2H, m), 4.23 (2H, q, $J=7.2 \mathrm{~Hz}), 3.88(1 \mathrm{H}, \mathrm{s}), 3.54$ (1H, d, $J=17.6 \mathrm{~Hz}), 3.39$ (1H, d, $J=17.6 \mathrm{~Hz}), 1.92-1.82(1 \mathrm{H}, \mathrm{m}), 1.72(2 \mathrm{H}, \mathrm{d}, J=6.0 \mathrm{~Hz}), 1.27(3 \mathrm{H}, \mathrm{t}, J=7.2 \mathrm{~Hz}), 1.01$ (3H, d, $J$ $=6.4 \mathrm{~Hz}), 0.91(3 \mathrm{H}, \mathrm{d}, J=6.4 \mathrm{~Hz}) ;{ }^{13} \mathrm{C} \mathrm{NMR}(100 \mathrm{MHz}): \delta 198.8,175.9,136.8,133.7,128.8$, 128.3, 75.5, 61.8, 48.3, 48.1, 24.6, 24.0, 23.8, 14.3; HRMS calcd for $\mathrm{C}_{16} \mathrm{H}_{22} \mathrm{O}_{4} \mathrm{Na}\left(\mathrm{M}+\mathrm{Na}^{+}\right)$: 301.1416, Found: 301.1416; $[\alpha]_{\mathrm{D}}{ }^{20}=-27.38\left(c=0.89, \mathrm{CHCl}_{3}\right)$.

Optical purity of the aldol adduct was established by chiral HPLC analysis: Chiracel OD (0.46 $\mathrm{x}$ $25 \mathrm{~cm})$, 95:5 hexanes:i-PrOH, $1.0 \mathrm{~mL} / \mathrm{min}, \lambda=254 \mathrm{~nm}, 87 \%$ ee.
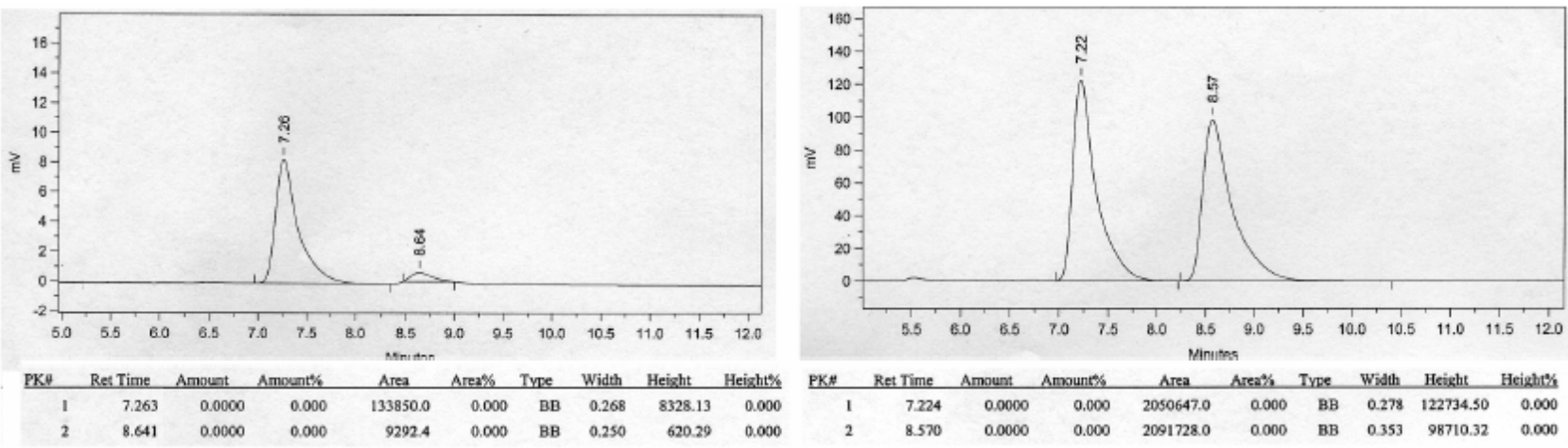

Ethyl-(S)-2-hydroxy-2-isopropyl-4-oxo-4-phenylbutanoate (Table 2, entry 4). The reaction mixture was allowed to stir at $-30{ }^{\circ} \mathrm{C}$ for $24 \mathrm{~h}$. Following purification, the aldol adduct was 
obtained as colorless oil. IR (neat): 3509 (br), 2966 (s), 1732 (s), 1686 (s), 1451 (m), 1350 (m), 1261 (s), 1216 (s), 1039 (s) cm ${ }^{-1}$; ${ }^{1} \mathrm{H}$ NMR (400 MHz): $\delta$ 7.95-7.92 (2H, m), 7.59 (1H, tt, $J=6.8$, $1.6 \mathrm{~Hz}), 7.48-7.44(2 \mathrm{H}, \mathrm{m}), 4.24(2 \mathrm{H}, \mathrm{dq}, J=7.2,1.2 \mathrm{~Hz}), 3.79(1 \mathrm{H}, \mathrm{s}), 3.51(1 \mathrm{H}, \mathrm{d}, J=17.2$ Hz), 3.45 (1H, d, $J=17.2 \mathrm{~Hz}), 2.00(1 \mathrm{H}, \mathrm{tt}, J=6.8,6.8 \mathrm{~Hz}), 1.26(3 \mathrm{H}, \mathrm{t}, J=3.6 \mathrm{~Hz}), 1.06$ (3H, d, $J=6.8 \mathrm{~Hz}), 0.96(3 \mathrm{H}, \mathrm{d}, J=6.8 \mathrm{~Hz}) ;{ }^{13} \mathrm{C} \mathrm{NMR}(100 \mathrm{MHz}): \delta 199.0,175.7,136.9,133.6$, 128.8, 128.2, 77.6, 61.7, 45.0, 35.9, 17.1, 16.7, 14.3; HRMS calcd for $\mathrm{C}_{15} \mathrm{H}_{20} \mathrm{O}_{4} \mathrm{Na}\left(\mathrm{M}+\mathrm{Na}^{+}\right)$: 287.1259, Found: 287.1252; $[\alpha]_{\mathrm{D}}{ }^{20}=-28.78\left(c=0.43, \mathrm{CHCl}_{3}\right)$.

Optical purity of the aldol adduct was established by chiral HPLC analysis: Chiracel OD (0.46 $\mathrm{x}$ $25 \mathrm{~cm}), 96: 4$ hexanes:i-PrOH, $1.0 \mathrm{~mL} / \mathrm{min}, \lambda=254 \mathrm{~nm}$; 95\% ee.
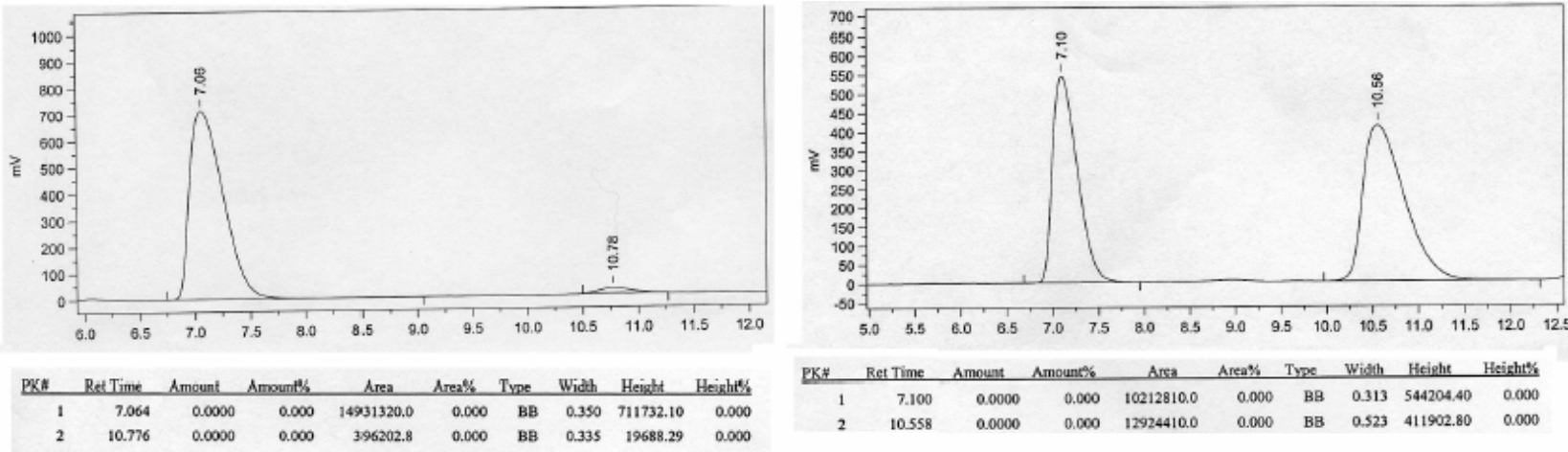

Ethyl-(S)-2-hydroxy-2-isopropyl-5,5-dimethyl-4-oxohexanoate (Table 2, entry 5). In a $16 \mathrm{x}$ $100 \mathrm{~mm}$ test tube under an atmosphere of $\mathrm{N}_{2}$ in a glove box, ${ }^{3} \mathrm{AgF}_{2}$, chiral ligand 5d, ethyl-3methyl-2-oxobutyrate, and tetrahydrofuran are combined as described in the general procedure. The test tube is sealed with a septum and teflon tape, removed from the glove box, and the mixture is allowed to stir for $20 \mathrm{~min}$. The solution is cooled to $-78{ }^{\circ} \mathrm{C}$ and enolsilane added as described in the general procedure. The reaction mixture is allowed to stir at $-15{ }^{\circ} \mathrm{C}$ for $48 \mathrm{~h}$. Following purification, the desired product is obtained as colorless oil. IR (neat): 3527 (br), 2967 (s), 2931 (s), 2873 (m), 1732 (s), 1710 (s), 1463 (m), 1394 (m), 1369 (m), 1268 (m), 1214 (s), 1191 (m), 1079 (m), 1048 (m) cm ${ }^{-1}$; ${ }^{1} \mathrm{H}$ NMR (400 MHz): $\delta 4.21$ (2H, q, J = $6.8 \mathrm{~Hz}$ ), 3.69 (1H, s), 3.02 (1H, d, $J=17.6 \mathrm{~Hz}), 2.92(1 \mathrm{H}, \mathrm{d}, J=17.6 \mathrm{~Hz}), 1.87$ (1H, qq, $J=6.8,6.8 \mathrm{~Hz}), 1.25$ $(3 \mathrm{H}, \mathrm{t}, J=6.8 \mathrm{~Hz}), 1.12(9 \mathrm{H}, \mathrm{s}), 0.96(3 \mathrm{H}, \mathrm{d}, J=6.8 \mathrm{~Hz}), 0.88(3 \mathrm{H}, \mathrm{d}, J=6.8 \mathrm{~Hz}) ;{ }^{13} \mathrm{C}$ NMR (100 MHz): $\delta 215.4,175.7,77.5,77.1,76.8,61.5,44.4,43.4,35.8,26.3,17.1$, 16.6, 14.3; HRMS calcd for $\mathrm{C}_{13} \mathrm{H}_{24} \mathrm{O}_{4} \mathrm{Na}\left(\mathrm{M}+\mathrm{Na}^{+}\right)$: 267.1572, Found: 267.1564; $[\alpha]_{\mathrm{D}}{ }^{20}=-18.79\left(c=0.45, \mathrm{CHCl}_{3}\right)$. Optical purity of the aldol adduct was established by chiral GLC analysis: Chiraldex GTA, $\left.100{ }^{\circ} \mathrm{C}, 15 \mathrm{psi}\right) ; 92 \%$ ee.

\footnotetext{
${ }^{3}$ For addition of the enolsilane derived from 3,3-dimethyl-2-butanone higher conversion is observed when reactions are prepared under an inert atmosphere in a glove box.
} 

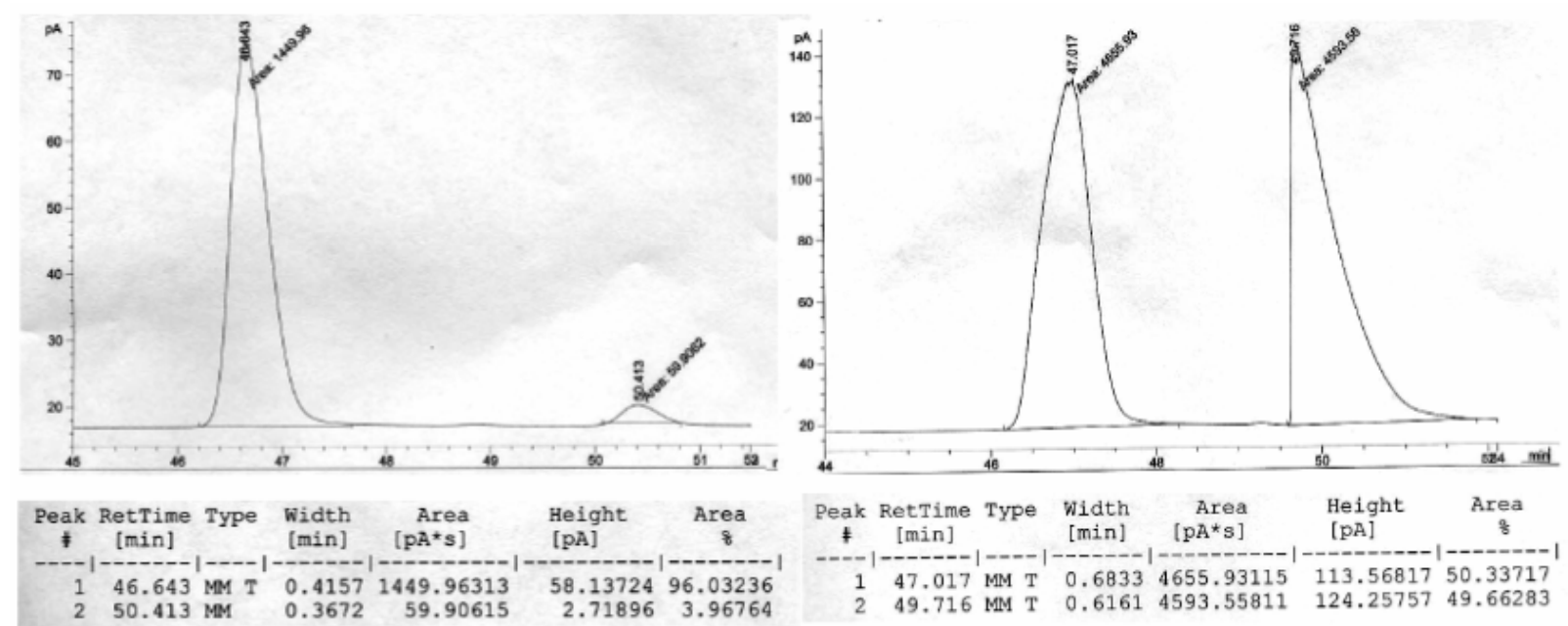

Ethyl-(S)-2-hydroxy-2-isopropyl-4-oxopentanoate (Table 2, entry 6). The mixture is allowed to stir at $-40{ }^{\circ} \mathrm{C}$ for $48 \mathrm{~h}$. Following purification, the desired product is obtained as colorless oil. IR (neat): 3503 (br), 2970 (s), 2879 (w), 1731 (s), 1363 (w), 1267 (w), 1213 (w), 1165 (w), 1131 (w), 1041 (w) cm ${ }^{-1}$; ${ }^{1} \mathrm{H}$ NMR (400 MHz): $\delta 4.23$ (2H, q, J = 7.2 Hz), $3.65(1 \mathrm{H}$, br s), 2.97 (1H, d, $J=17.6 \mathrm{~Hz}), 2.87(1 \mathrm{H}, \mathrm{d}, J=17.6 \mathrm{~Hz}), 2.16(3 \mathrm{H}, \mathrm{s}), 1.91-1.84(1 \mathrm{H}, \mathrm{m}), 1.28(3 \mathrm{H}, \mathrm{t}, J=7.2 \mathrm{~Hz})$, 0.95 (3H, d, $J=7.2 \mathrm{~Hz}), 0.87$ (3H, d, $J=7.2 \mathrm{~Hz}) ;{ }^{13} \mathrm{C}$ NMR (100 MHz): $\delta$ 207.8, 175.5, 77.6, 61.8, 49.4, 35.7, 31.0, 17.0, 16.5, 14.3; HRMS calcd for $\mathrm{C}_{10} \mathrm{H}_{18} \mathrm{O}_{4} \mathrm{Na}\left(\mathrm{M}+\mathrm{Na}^{+}\right)$: 225.1103, Found: 225.1113; $[\alpha]_{\mathrm{D}}{ }^{20}=-20.62\left(c=0.63, \mathrm{CHCl}_{3}\right)$.

Optical purity of the aldol adduct was established by chiral GLC analysis: Chiraldex GTA, $\left.100{ }^{\circ} \mathrm{C}, 15 \mathrm{psi}\right) ; 88 \%$ ee.
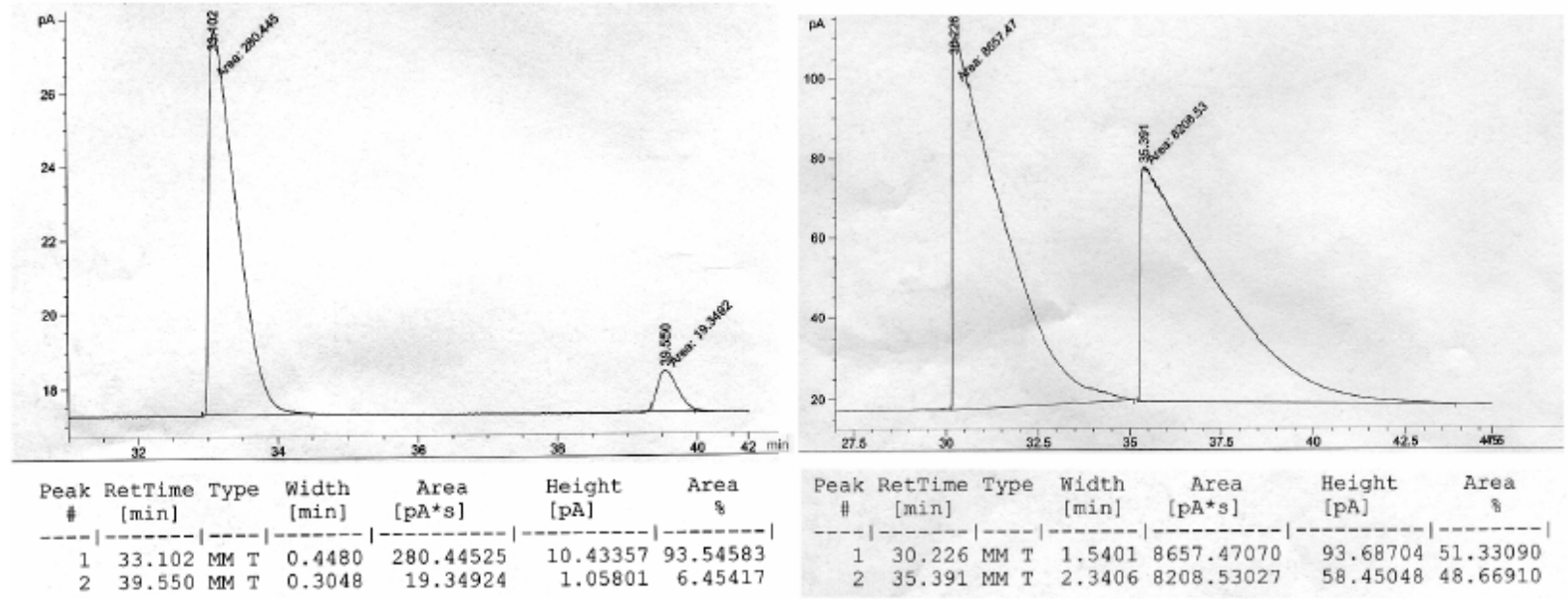

Ethyl-(S)-2-cyclohexyl-2-hydroxy-4-oxo-4-phenylbutanoate (Table 2, entry 7). The mixture is allowed to stir at $-30{ }^{\circ} \mathrm{C}$ for $24 \mathrm{~h}$. Following purification, the desired product is obtained as colorless oil. IR (neat): 3521 (br), 2930 (s), 2855 (m), 1732 (s), 1686 (s), 1596 (w), 1449 (s), 1357 (m), 1218 (s) cm ${ }^{-1}$; ${ }^{1} \mathrm{H}$ NMR (400 MHz): $\delta$ 7.95-7.92 (2H, m), $7.57(1 \mathrm{H} \mathrm{tt}, J=6.8,1.2 \mathrm{~Hz})$, 7.48-7.44 (2H, m), 4.24 (2H, q, $J=1.8 \mathrm{~Hz}), 3.81(1 \mathrm{H}$, br s), $3.52(1 \mathrm{H}, \mathrm{d}, J=17.2 \mathrm{~Hz}), 3.46(1 \mathrm{H}$, d, $J=17.2 \mathrm{~Hz}), 1.91-1.78$ (3H, m), 1.69-1.57 (3H, m), 1.32-1.16 (8H, m); ${ }^{13} \mathrm{C} \mathrm{NMR} \mathrm{(100} \mathrm{MHz):}$ 8199.2, 175.6, 137.0, 133.6, 128.8, 128.3, 77.8, 61.7, 45.7, 44.8, 27.2, 26.7, 26.5, 26.4, 26.3, 
14.3; HRMS calcd for $\mathrm{C}_{18} \mathrm{H}_{25} \mathrm{O}_{4}\left(\mathrm{M}+\mathrm{H}^{+}\right): 305.1753$, Found: $305.1739 ;[\alpha]_{\mathrm{D}}{ }^{20}=-31.54 \quad(c=$ $\left.0.77, \mathrm{CHCl}_{3}\right)$.

Optical purity of the aldol adduct was established by diastereoselective reduction $\left(\mathrm{BH}_{3} \bullet \mathrm{THF}\right.$, $22{ }^{\circ} \mathrm{C}, 15 \mathrm{~min} ; \mathrm{NaBH}_{4}, 4 \mathrm{~h}$ ) of the ketone, followed by acid-catalyzed lactonization (acidic workup: addition of a $20 \%$ solution of $p-\mathrm{TsOH}$ in $\mathrm{MeOH}$ at $22{ }^{\circ} \mathrm{C}$ for $1 \mathrm{~h}$; filtration and removal of solvents in vacuo). Optical purity of the derived lactone was established by chiral HPLC analysis in comparison to an authentic racemic sample: Chiracel OD (0.46 x $25 \mathrm{~cm}), 98: 2$ hexanes:i-PrOH, $1.0 \mathrm{~mL} / \mathrm{min}, \lambda=254 \mathrm{~nm}$; 95\% ee.
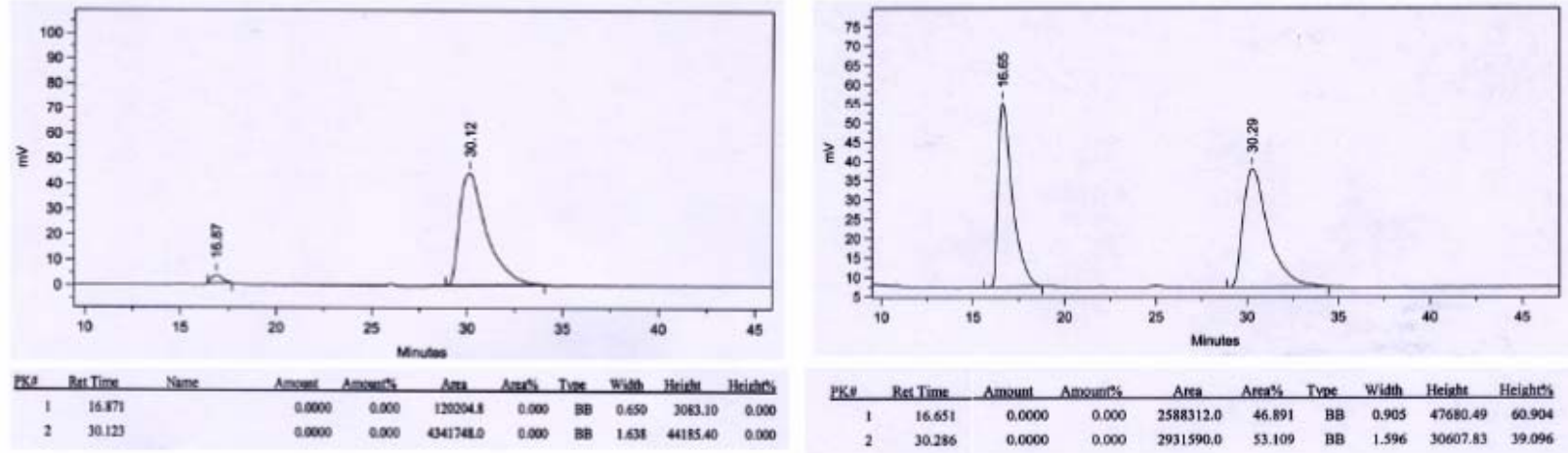

Ethyl-(S)-2-cyclohexyl-2-hydroxy-4-oxopentanoate (Table 2, entry 8). The mixture is allowed to stir at $-30{ }^{\circ} \mathrm{C}$ for $24 \mathrm{~h}$. Following purification, the desired product is obtained as colorless oil. IR (neat): 3509 (br), 2931 (s), 2854 (m), 1728 (s), 1451 (w), 1364 (m), 1267 (w), 1212 (s), 1113 (m) $\mathrm{cm}^{-1} ;{ }^{1} \mathrm{H}$ NMR (400 MHz): $\delta 4.33(2 \mathrm{H}, \mathrm{q}, J=7.2 \mathrm{~Hz}), 3.66(1 \mathrm{H}, \mathrm{s}), 2.97(1 \mathrm{H}, \mathrm{d}, J=17.2$ Hz), 2.89 (1H, d, $J=17.2 \mathrm{~Hz}), 2.15$ (3H, s), 1.81-1.75 (3H, m), 1.66-1.63 (1H, m), 1.55-1.49 (2H, m), 1.28 (3H, t, $J=7.2 \mathrm{~Hz}), 1.27-1.07$ (5H, m); ${ }^{13} \mathrm{C}$ NMR (100 MHz): $\delta$ 208.0, 175.4, 77.7, 61.8, 45.2, 45.5, 31.0, 27.0, 26.5, 26.4, 26.3, 26.2, 14.3; HRMS calcd for $\mathrm{C}_{13} \mathrm{H}_{22} \mathrm{O}_{4}$ : 242.1518, Found: 242.1520; $[\alpha]_{\mathrm{D}}{ }^{20}=-16.07\left(c=0.87, \mathrm{CHCl}_{3}\right)$.

Optical purity of the aldol adduct was established by chiral GLC analysis: Chiraldex GTA, $\left.120{ }^{\circ} \mathrm{C}, 15 \mathrm{psi}\right) ; 90 \%$ ee.
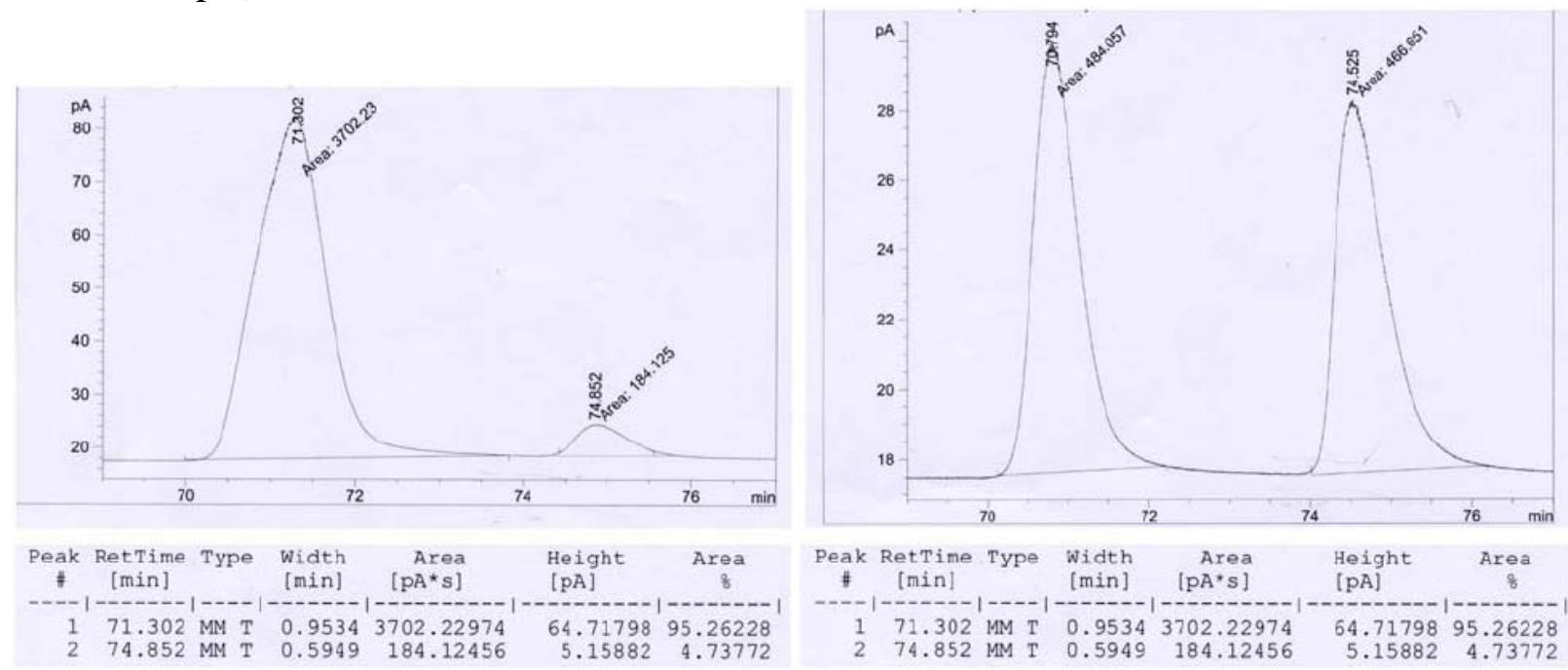
Ethyl-(S)-2-cyclopropyl-2-hydroxy-4-oxo-4-phenylbutanoate (Table 2, entry 9). The mixture is allowed to stir at $-40{ }^{\circ} \mathrm{C}$ for $48 \mathrm{~h}$. Following purification, the desired product is obtained as colorless oil. IR (neat): 3503 (br), 2974 (w), 1732 (s), 1686 (s), 1449 (m), 1356 (m), 1276 (m), 1210 (s), 1051 (m) cm ${ }^{-1}$; ${ }^{1} \mathrm{H}$ NMR (400 MHz): $\delta$ 7.96-7.94 (2H, m), $7.58(1 \mathrm{H}, \mathrm{tt}, J=7.2,1.2 \mathrm{~Hz})$, 7.48-7.44 (2H, m), 4.28 (2H, q, $J=7.2 \mathrm{~Hz}), 3.63(1 \mathrm{H}, \mathrm{dd}, J=17.6,0.4 \mathrm{~Hz}), 3.53(1 \mathrm{H}, \mathrm{d}$, $J=17.6 \mathrm{~Hz}$ ), 3.48 (1H, s), 1.27 (3H, t, $J=7.2 \mathrm{~Hz}), 1.21-1.13(1 \mathrm{H}, \mathrm{m}), 0.65-0.54$ (2H, m), 0.490.42 (1H, m), 0.39-0.33 (1H, m); ${ }^{13} \mathrm{C}$ NMR (100 MHz): $\delta 198.3,175.9,136.7,133.6,128.8$, 128.3, 72.5, 62.0, 47.7, 18.5, 14.3, 0.35, -0.23; HRMS calcd for $\mathrm{C}_{15} \mathrm{H}_{18} \mathrm{O}_{4} \mathrm{Na}\left(\mathrm{M}+\mathrm{Na}^{+}\right)$: 285.1103, Found: 285.1087; $[\alpha]_{\mathrm{D}}{ }^{20}=-33.19\left(c=1.04, \mathrm{CHCl}_{3}\right)$.

Optical purity of the aldol adduct was established by chiral HPLC analysis: Chiracel OD (0.46 $\mathrm{x}$ $25 \mathrm{~cm})$, 95:5 hexanes:i-PrOH, $1.0 \mathrm{~mL} / \mathrm{min}, \lambda=254 \mathrm{~nm} ; 96 \%$ ee.
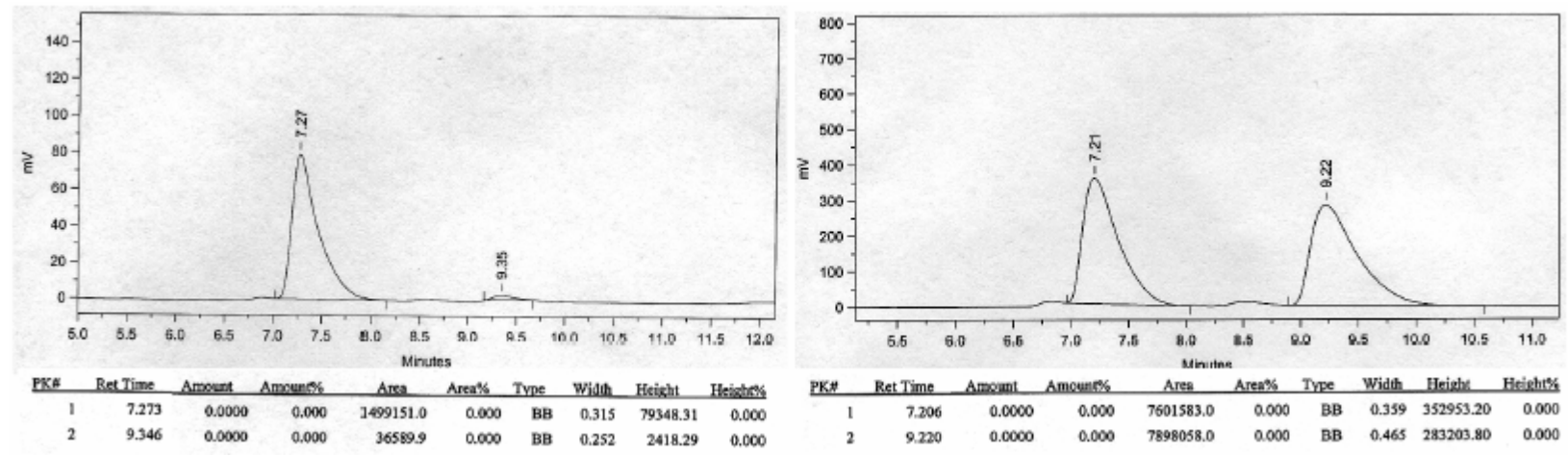

Ethyl-(S)-2-hydroxy-3-methyl-2-(2-oxo-2-phenylethyl)but-3-enoate (Table 2, entry 10). The mixture is allowed to stir at $-40{ }^{\circ} \mathrm{C}$ for $48 \mathrm{~h}$. Following purification, the desired product is obtained as colorless oil. IR (neat): 3509 (br), 2980 (m), 2936 (w), 1729 (s), 1686 (s), 1449 (m), 1282 (m), 1283 (m), 1211 (s), 1069 (m), 690 (m); ${ }^{1} \mathrm{H}$ NMR (400 MHz): $\delta$ 7.97-7.94 (2H, m), $7.59(1 \mathrm{H}, \mathrm{tt}, J=7.2,1.2 \mathrm{~Hz}), 7.50-7.45(2 \mathrm{H}, \mathrm{m}), 5.30-5.29(1 \mathrm{H}, \mathrm{m}), 5.07-5.06(1 \mathrm{H}, \mathrm{m}), 4.26(2 \mathrm{H}$, dq, $J=7.2,1.2 \mathrm{~Hz}), 3.77(1 \mathrm{H}, \mathrm{d}, J=17.2 \mathrm{~Hz}), 3.46(1 \mathrm{H}, \mathrm{d}, J=17.2 \mathrm{~Hz}), 1.88-1.87$ (3H, m), 1.27 $(3 \mathrm{H}, \mathrm{t}, J=7.2 \mathrm{~Hz}) ;{ }^{13} \mathrm{C} \mathrm{NMR}(100 \mathrm{MHz}): \delta 199.0,173.9,144.1,136.7,133.8,128.8,128.3$, 113.4, 77.6, 62.2, 45.5, 19.0, 14.2; HRMS calcd for $\mathrm{C}_{15} \mathrm{H}_{18} \mathrm{O}_{4} \mathrm{Na}\left(\mathrm{M}+\mathrm{Na}^{+}\right)$: 285.1103, Found: 285.1099; $[\alpha]_{\mathrm{D}}{ }^{20}=-14.67\left(c=0.80, \mathrm{CHCl}_{3}\right)$.

Optical purity of the aldol adduct was established by chiral HPLC analysis: Chiracel OD (0.46 $\mathrm{x}$ $25 \mathrm{~cm})$, 95:5 hexanes:i-PrOH, $1.0 \mathrm{~mL} / \mathrm{min}, \lambda=254 \mathrm{~nm}$; $90 \%$ ee.
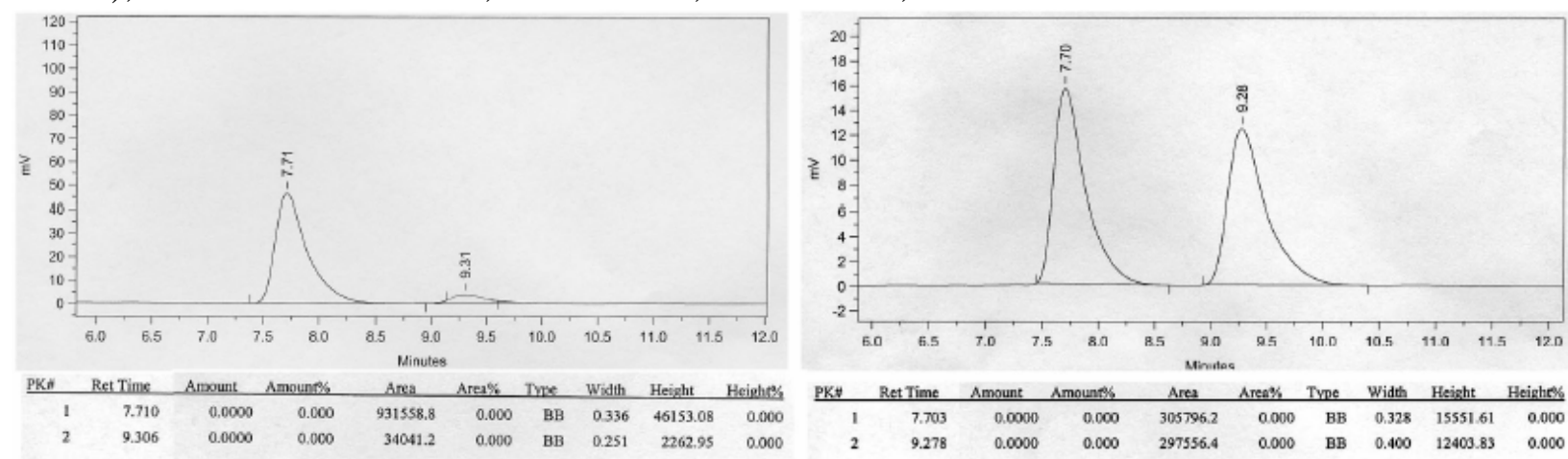
Ethyl-(S)-2-hydroxy-4-oxo-2,4-diphenylbutanoate (Table 2, entry 11). The mixture is allowed to stir at $-30{ }^{\circ} \mathrm{C}$ for $24 \mathrm{~h}$. Following purification, the desired product is obtained as colorless oil. IR (neat): 3515 (br), 3055 (w), 2974 (m), 1730 (s), 1683 (s), 1448 (m), 1267 (m), 1213 (s), 1180 (m), 1060 (m) cm ${ }^{-1}$; ${ }^{1} \mathrm{H}$ NMR (400 MHz): $87.98-7.95$ (2H, m), 7.69-7.67 (2H, m), $7.60(1 \mathrm{H}, \mathrm{tt}, J=6.8,1.2 \mathrm{~Hz}), 7.50-7.45(2 \mathrm{H}, \mathrm{m}), 7.42-7.38(2 \mathrm{H}, \mathrm{m}), 7.36-7.32(1 \mathrm{H}, \mathrm{m}), 4.51(1 \mathrm{H}$, s), $4.26(2 \mathrm{H}, \mathrm{q}, J=7.2 \mathrm{~Hz}), 4.07(1 \mathrm{H}, \mathrm{d}, J=17.6 \mathrm{~Hz}), 3.58(1 \mathrm{H}, \mathrm{d}, J=17.6 \mathrm{~Hz}), 1.25(3 \mathrm{H}, \mathrm{t}, J=$ 7.2 Hz); ${ }^{13} \mathrm{C}$ NMR (100 MHz): $\delta 198.7,174.1,140.8,136.4,133.8,128.8,128.6,128.3,128.2$, 125.2, 76.5, 62.3, 49.1, 14.1; HRMS calcd for $\mathrm{C}_{18} \mathrm{H}_{18} \mathrm{O}_{4} \mathrm{Na}\left(\mathrm{M}+\mathrm{Na}^{+}\right)$: 321.1103, Found: 321.1110; Anal Calcd for $\mathrm{C}_{18} \mathrm{H}_{18} \mathrm{O}_{4}$ : C, 72.47; H, 6.08; Found: C, 72.51; H, 6.36; $[\alpha]_{\mathrm{D}}{ }^{20}=-36.79$ $\left(c=0.90, \mathrm{CHCl}_{3}\right)$.

Optical purity of the aldol adduct was established by chiral HPLC analysis: Chiracel OD (0.46 $\mathrm{x}$ $25 \mathrm{~cm}$ ), 98:2 hexanes:i-PrOH, $0.5 \mathrm{~mL} / \mathrm{min}, \lambda=254 \mathrm{~nm}$; $60 \%$ ee.
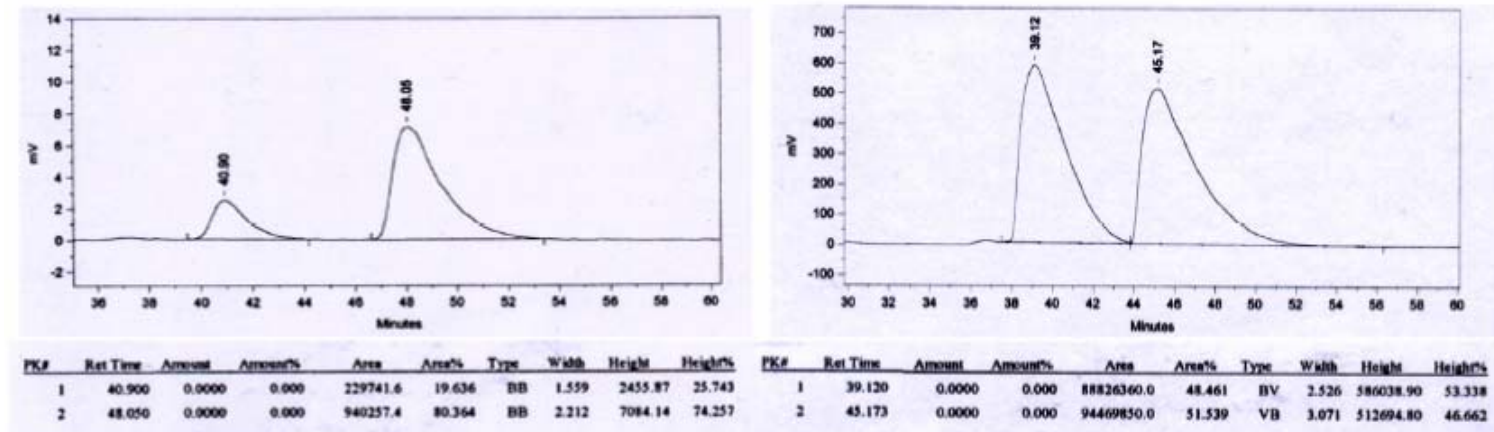

Ethyl-(R)-2-hydroxy-4-oxo-4-phenyl-2-(thiophen-2-yl)butanoate (Table 2, entry 12). The mixture is allowed to stir at $-30{ }^{\circ} \mathrm{C}$ for $48 \mathrm{~h}$. Following purification, the desired product is obtained as pale yellow oil. IR (neat): 3483 (br), 3062 (w), 2980 (m), 1737 (s), 1680 (s), 1597 (s), 1449 (m), 1352 (m), 1266 (m), 1214 (s), 1115 (m), 1061 (m) cm ${ }^{-1}$; ${ }^{1} \mathrm{H}$ NMR (400 MHz): $\delta$ 7.97-7.95 (2H, m), $7.60(1 \mathrm{H}, \mathrm{tt}, J=6.8,1.6 \mathrm{~Hz}), 7.50-7.46(2 \mathrm{H}, \mathrm{m}), 7.29(1 \mathrm{H}, \mathrm{dd}, J=5.3,1.2$ Hz), 7.15 (1H, dd, $J=4.0,1.2 \mathrm{~Hz}), 7.01(1 \mathrm{H}, \mathrm{dd}, J=5.3,4.0 \mathrm{~Hz}), 4.60(1 \mathrm{H}, \mathrm{s}), 4.29(2 \mathrm{H}, \mathrm{q}, J=$ $7.2 \mathrm{~Hz}), 4.02(1 \mathrm{H}, \mathrm{d}, J=17.6 \mathrm{~Hz}), 3.78(1 \mathrm{H}, \mathrm{d}, J=17.6 \mathrm{~Hz}), 1.28(3 \mathrm{H}, \mathrm{t}, J=7.2 \mathrm{~Hz})$; ${ }^{13} \mathrm{C} \mathrm{NMR}$ (100 MHz): $\delta 197.7,173.3,145.7,136.4,134.0,128.9,128.4,127.3,125.7,124.1,75.4,62.8$, 49.8, 14.2; HRMS calcd for $\mathrm{C}_{16} \mathrm{H}_{16} \mathrm{O}_{4} \mathrm{SNa}\left(\mathrm{M}+\mathrm{Na}^{+}\right)$: 327.0667, Found: 327.0676; $[\alpha]_{\mathrm{D}}{ }^{20}=-$ $33.59\left(c=0.77, \mathrm{CHCl}_{3}\right)$.

Optical purity of the aldol adduct was established by chiral HPLC analysis: Chiracel AS (0.46 $\mathrm{x}$ $25 \mathrm{~cm}), 95: 5$ hexanes:i-PrOH, $1.0 \mathrm{~mL} / \mathrm{min}, \lambda=254 \mathrm{~nm}$; $72 \%$ ee.
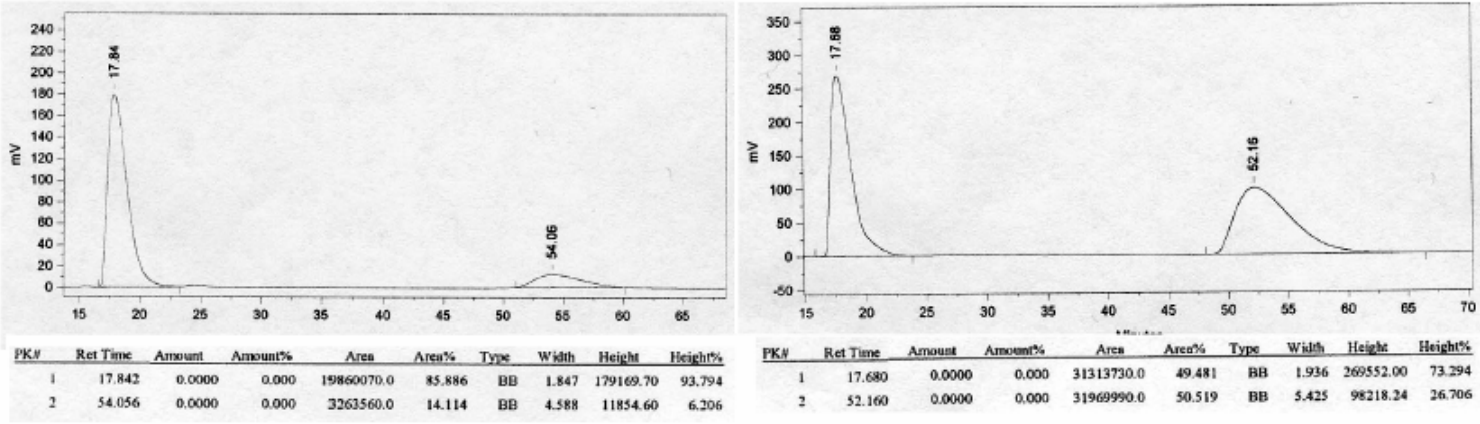
Methyl-(R)-5-oxo-2-(2-oxo-2-phenylethyl)tetrahydrofuran-2-carboxylate (eq 1). The mixture is allowed to stir at $-30{ }^{\circ} \mathrm{C}$ for $24 \mathrm{~h}$ after which $1 \mathrm{~mL}$ of a $2 \mathrm{~N}$ solution of $\mathrm{HCl}$ is added; the resulting solution is allowed to stir for $4 \mathrm{~h}$. The mixture is washed with $\mathrm{Et}_{2} \mathrm{O}$, as described in the general procedure. The purified product is obtained as a white solid. m. p. $=70.5{ }^{\circ} \mathrm{C}$; IR (neat): 1785 (s), 1746 (m), 1687 (m), 1451 (w), 1369 (w), 1173 (s), 1064 (s); ${ }^{1} \mathrm{H}$ NMR (400 MHz): $\delta 7.93(2 \mathrm{H}, \mathrm{d}, J=7.6 \mathrm{~Hz}), 7.60(1 \mathrm{H}, \mathrm{t}, J=7.2 \mathrm{~Hz}), 7.48(2 \mathrm{H}, \mathrm{t}, J=7.6 \mathrm{~Hz}), 3.83(1 \mathrm{H}, \mathrm{d}, J=17.6$ $\mathrm{Hz}), 3.82(3 \mathrm{H}, \mathrm{s}), 3.68(1 \mathrm{H}, \mathrm{d}, J=17.6 \mathrm{~Hz}), 2.84-2.59(3 \mathrm{H}, \mathrm{m}), 2.38-2.27(1 \mathrm{H}, \mathrm{m}) ;{ }^{13} \mathrm{C}$ NMR (100 MHz): $\delta$ 195.2, 175.9, 171.3, 136.2, 134.0, 129.0, 128.3, 83.7, 53.4, 46.1, 31.4, 28.1; HRMS calcd for $\mathrm{C}_{14} \mathrm{H}_{14} \mathrm{O}_{5} \mathrm{Na}\left(\mathrm{M}+\mathrm{Na}^{+}\right)$: 285.0739, Found: 285.0758; $[\alpha]_{\mathrm{D}}{ }^{20}=+11.96(c=1.02$, $\mathrm{CHCl}_{3}$ ).

Representative experimental procedure for enantioselective Ag-catalyzed hetero-DielsAlder (HDA) reactions of $\boldsymbol{\alpha}$-ketoesters: A 16 x $100 \mathrm{~mm}$ test tube equipped with a magnetic stir bar is charged with $\mathrm{AgF}_{2}$ (1.8 mg, $0.012 \mathrm{mmol}$ ), chiral ligand 5d (5.2 mg, $0.012 \mathrm{mmol}$ ) and tetrahydrofuran $(0.40 \mu \mathrm{L})$ is added. The resulting solution is allowed to stir while ethyl-3methyl-2-oxobutyrate $(17 \mu \mathrm{L}, 0.12 \mathrm{mmol})$ is added followed by the addition of $0.40 \mu \mathrm{L}$ of tetrahydrofuran. The tube is capped with a septum, purged with $\mathrm{N}_{2}$, and allowed to stir for 20 min, after which the solution is allowed to cool to $-78{ }^{\circ} \mathrm{C}$, and trans-1-methoxy-3(trimethylsiloxy)1,3-butadiene $(27 \mu \mathrm{L}, 0.14 \mathrm{mmol})$ dissolved in THF $(0.40 \mathrm{~mL})$ is added in a dropwise fashion (syringe). The test tube is sealed with teflon tape and warmed to $\quad-30{ }^{\circ} \mathrm{C}$ in a circulating bath. The mixture is allowed to stir for $24 \mathrm{~h}$ at $-30{ }^{\circ} \mathrm{C}$, after which $1 \mathrm{~mL}$ of a $2.0 \mathrm{M}$ aqueous solution of $\mathrm{HCl}$ is added and the mixture is allowed to warm to $22{ }^{\circ} \mathrm{C}$ and stir for $1 \mathrm{~h}$. The aqueous layer is washed with $\mathrm{Et}_{2} \mathrm{O}(3 \times 1.0 \mathrm{~mL})$ and the combined organic layers are filtered through silica and concentrated under reduced pressure to afford pale yellow oil, which is purified by silica gel chromatography $\left(5: 1\right.$ pentane:Et $\left.{ }_{2} \mathrm{O}\right)$ to deliver the desired unsaturated pyranone as a colorless oil (17 $\mathrm{mg}, 0.08 \mathrm{mmol}, 66 \%$ yield).

Ethyl-(S)-2-isopropyl-4-oxo-3,4-dihydro-2H-pyran-2-carboxylate (eq 3, R = i-Pr). The mixture is allowed to stir at $-30{ }^{\circ} \mathrm{C}$ for $24 \mathrm{~h}$. Following purification, the desired cycloadduct is obtained as colorless oil. IR (neat): 2967 (m), 2817 (s), 2848 (m), 1734 (s), 1682 (s), 1597 (s), 1463 (m), 1400 (m), 1274 (s), 1218 (m), 1174 (m) cm ${ }^{-1}$; ${ }^{1} \mathrm{H}$ NMR (400 MHz): $\delta 7.37$ (1H, d, $J=$ $6.1 \mathrm{~Hz}), 5.40$ (1H, dd, $J=6.1,1.2 \mathrm{~Hz}), 4.22(2 \mathrm{H}, \mathrm{tt}, J=7.2,7.2 \mathrm{~Hz}), 2.89(1 \mathrm{H}, \mathrm{dd}, J=16.8,1.2$ $\mathrm{Hz}), 2.76(1 \mathrm{H}, \mathrm{d}, J=16.8 \mathrm{~Hz}), 2.22(1 \mathrm{H}, \mathrm{tt}, J=6.8,6.8 \mathrm{~Hz}), 1.26(3 \mathrm{H}, \mathrm{t}, J=6.8 \mathrm{~Hz}), 1.02(3 \mathrm{H}$, d, $J=6.8 \mathrm{~Hz}), 1.01(3 \mathrm{H}, \mathrm{d}, J=6.8 \mathrm{~Hz}) ;{ }^{13} \mathrm{C} \mathrm{NMR}(100 \mathrm{MHz}): \delta 190.8,170.6,162.5,107.6,88.7$, 62.3, 40.7, 34.9, 16.9, 16.7, 14.3; HRMS calcd for $\mathrm{C}_{11} \mathrm{H}_{17} \mathrm{O}_{4}\left(\mathrm{M}+\mathrm{H}^{+}\right)$: 213.1120, Found: 213.1127; $[\alpha]_{\mathrm{D}}{ }^{20}=-125.15\left(c=0.93, \mathrm{CHCl}_{3}\right)$.

Optical purity of the cycloadduct was established by chiral GLC analysis: Chiraldex GTA, $\left.130{ }^{\circ} \mathrm{C}, 15 \mathrm{psi}\right) ; 90 \%$ ee. 

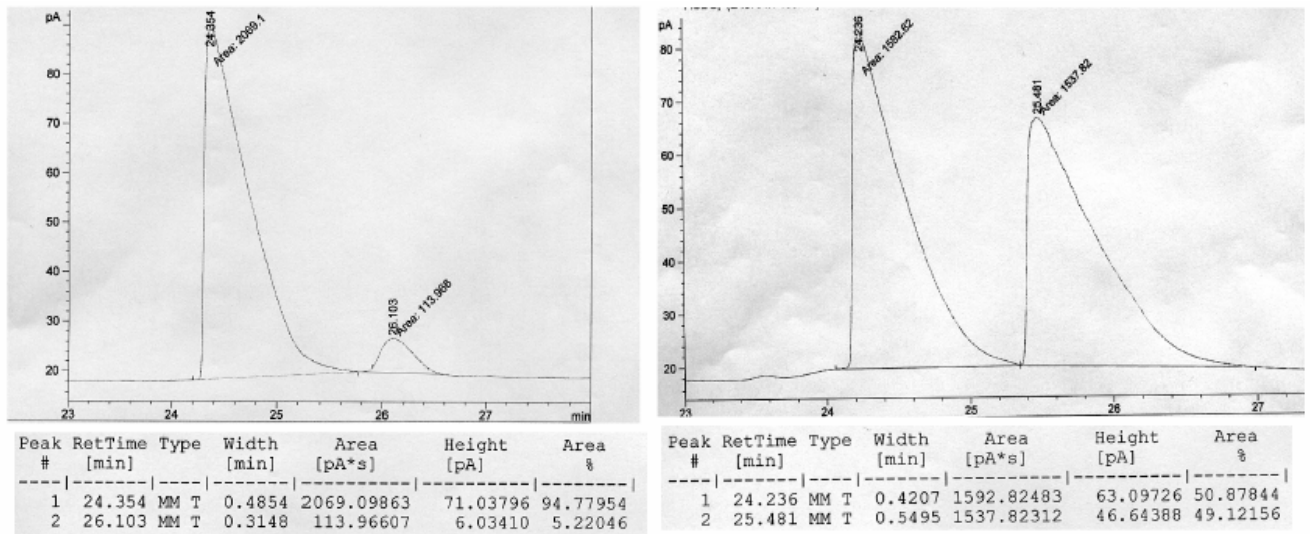

Ethyl-(S)-2-(2-cyclohexyl-4-oxo-3,4-dihydro-2H-pyran-2-yl)-2-oxoacetate (eq 3, R = Cy). The mixture is allowed to stir at $-30{ }^{\circ} \mathrm{C}$ for $24 \mathrm{~h}$. Following purification, the desired cycloadduct is obtained as colorless oil. IR (neat): 2929 (s), 2854 (m), 1733 (s), 1684 (s), 1597 (s), 1451 (w), 1401 (m), 1273 (s), 1221 (m), 1181 (m), 1026 (m) cm ${ }^{-1}$; ${ }^{1} \mathrm{H}$ NMR (400 MHz): $\delta 7.50(1 \mathrm{H}, \mathrm{d}, J=$ $6.0 \mathrm{~Hz}$ ), 5.39 (1H, dd, $J=6.0,1.2 \mathrm{~Hz}), 4.21(2 \mathrm{H}, \mathrm{q}, J=6.8 \mathrm{~Hz}), 2.87$ (1H, dd, $J=16.4,1.2 \mathrm{~Hz})$, $2.79(1 \mathrm{H}, \mathrm{d}, J=16.4 \mathrm{~Hz}), 1.90-1.68(6 \mathrm{H}, \mathrm{m}), 1.31-1.03(8 \mathrm{H}, \mathrm{m}) ;{ }^{13} \mathrm{C}$ NMR $(100 \mathrm{MHz}): \delta 191.0$, 170.7, 162.5, 107.6, 88.7, 62.3, 44.6, 40.8, 27.1, 26.9, 26.3, 26.2, 14.4; HRMS calcd for $\mathrm{C}_{14} \mathrm{H}_{20} \mathrm{O}_{4}: 252.1362$, Found: 252.1358; $[\alpha]_{\mathrm{D}}{ }^{20}=-106.34^{\circ}\left(c=0.04, \mathrm{CHCl}_{3}\right)$.

Optical purity of the cycloadduct was established by chiral GLC analysis: Chiraldex GTA, $\left.140{ }^{\circ} \mathrm{C}, 15 \mathrm{psi}\right) ; 90 \%$ ee.
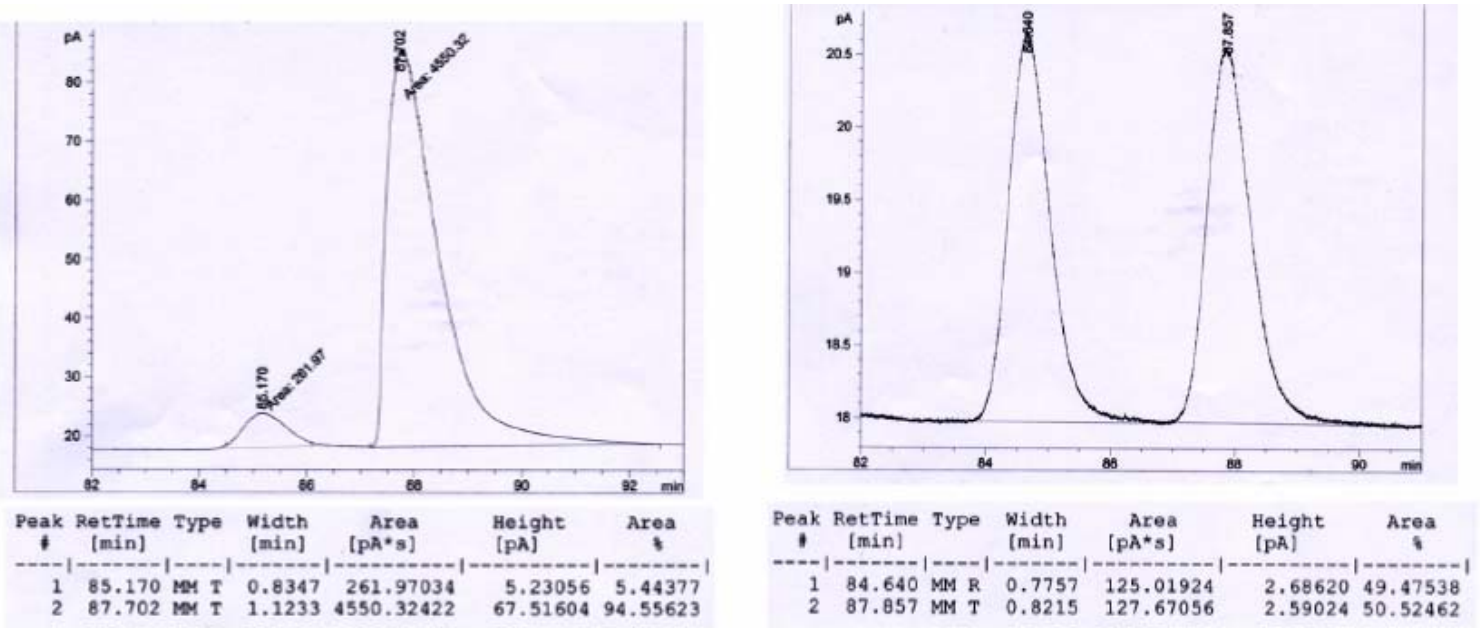

Proof of absolute stereochemistry. The spectral data for methyl-(R)-2-hydroxy-2-methyl-4oxo-4-phenylbutanoate are consistent with those reported in the literature. ${ }^{4}$ Moreover, as summarized in the Table below, the optical rotation measurements correspond to the $(R)$ enantiomer being formed as the major enantiomer with chiral ligand $\mathbf{5 d}$.

4) (a) Evans, D. A.; Burgey, C. S.; Kozlowski, M. C.; Tregay, S. W. J. Am. Chem. Soc. 1999, 121, 686-699. (b) Bolm, C.; Langner, M. Angew. Chem., Int. Ed. 2004, 43, 5984-5987. 
Table. Comparison of Specific Rotation Values

\begin{tabular}{cccc}
\hline study & $\begin{array}{c}\text { product } \\
\text { ee (\%) }\end{array}$ & specific rotation $\left[(\alpha)_{D}\right]$ & $\begin{array}{c}\text { absolute } \\
\text { configuration }\end{array}$ \\
\hline Evans (ref 4a) & 99 & $+84.4\left(c=3.5, \mathrm{CHCl}_{3}\right)$ & $\mathrm{S}$ \\
Bolm (ref 4b) & 98 & $-86.7\left(c=1.0, \mathrm{CHCl}_{3}\right)$ & $R$ \\
this study & 60 & $-39.6\left(c=3.5, \mathrm{CHCl}_{3}\right)$ & $R$ \\
\hline
\end{tabular}
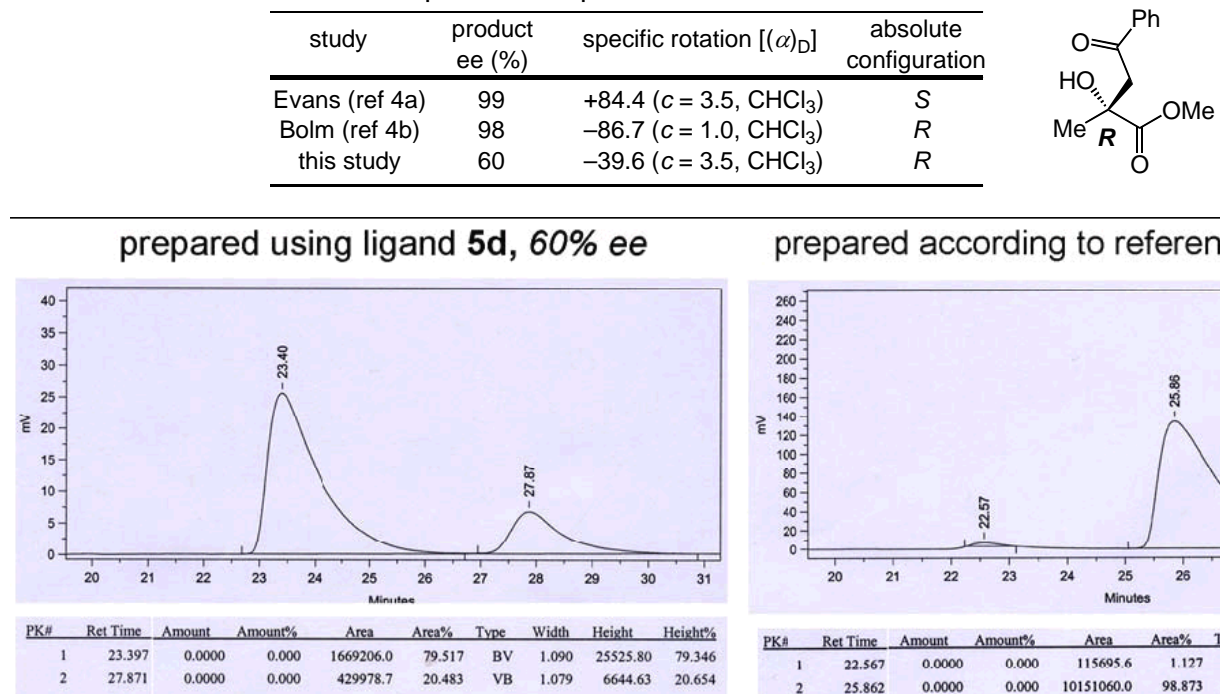

prepared according to reference $4 a, 98 \%$ ee

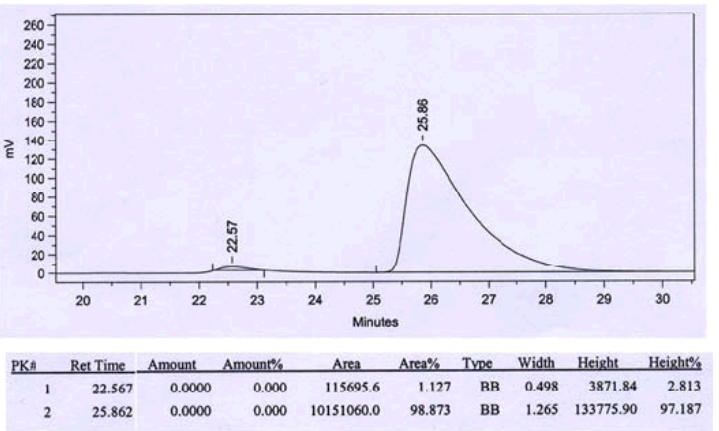

A sample of optically enriched methyl-2-hydroxy-2-methyl-4-oxo-4-phenylbutanoate was prepared through the use of $\mathrm{Cu}(\mathrm{OTf})_{2}$ and $(S, S)$-bis(tert-butyloxazoline), according to procedures described by Evans in reference 4a. Comparison of chiral HPLC data vs the optically enriched sample of the same compound obtained through Ag-catalyzed aldol addition confirms enrichment of the opposite enantiomer and the aforementioned stereochemical assignment (Chiracel OD (0.46 x $25 \mathrm{~cm}$ ), 98:2 hexanes:i-PrOH, $1.0 \mathrm{~mL} / \mathrm{min}, \quad \lambda=254 \mathrm{~nm}$; $(R)$ enantiomer $t_{r}=23.4 \mathrm{~min},(S)$ enantiomer $\mathrm{t}_{r}=27.9 \mathrm{~min}$.

Methyl-(R)-2-hydroxy-2-methyl-4-oxo-4-phenylbutanoate. The mixture is allowed to stir at $-30{ }^{\circ} \mathrm{C}$ for $24 \mathrm{~h}$. Following purification, the aldol adduct is obtained as a colorless oil. IR (neat): 3521 (br), 2980 (m), 2955 (m), 1740 (s), 1682 (s), 1450 (m), 1216 (s), 1114 (m) cm ${ }^{-1}$; ${ }^{1} \mathrm{H}$ NMR (400 MHz): $\delta$ 7.94-7.91 (2H, m), 7.58 (1H t, $J=7.2 \mathrm{~Hz}$ ), 7.48-7.44 (2H, m), 3.97 (1H, br s), 3.76 (3H, s), $3.66(1 \mathrm{H}, \mathrm{d}, J=17.6 \mathrm{~Hz}), 3.34(1 \mathrm{H}, \mathrm{d}, J=17.6 \mathrm{~Hz}), 1.50(3 \mathrm{H}, \mathrm{s}) ;{ }^{13} \mathrm{C} \mathrm{NMR}(100 \mathrm{MHz})$ : $\delta 202.8,199.12$, 136.5, 134.0, 128.9, 128.4, 72.9, 53.0, 48.2, 26.7; HRMS calcd for $\mathrm{C}_{12} \mathrm{H}_{14} \mathrm{O}_{4} \mathrm{Na}$ $\left(\mathrm{M}+\mathrm{Na}^{+}\right)$: 245.0790, Found: 245.0806; $[\alpha]_{\mathrm{D}}{ }^{20}=-39.58\left(c=3.5, \mathrm{CHCl}_{3}\right)$.

Optical purity of the aldol adduct was established by chiral HPLC analysis: Chiracel OD (0.46 $\mathrm{x}$ $25 \mathrm{~cm}$ ), 98:2 hexanes:i-PrOH, $1.0 \mathrm{~mL} / \mathrm{min}, \lambda=254 \mathrm{~nm} ; 60 \%$ ee.
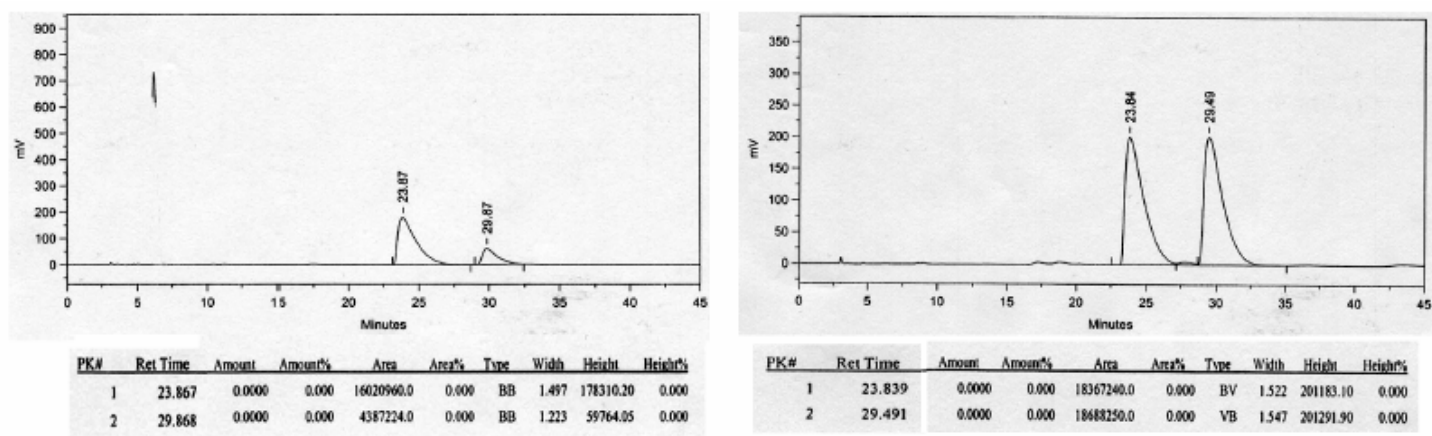


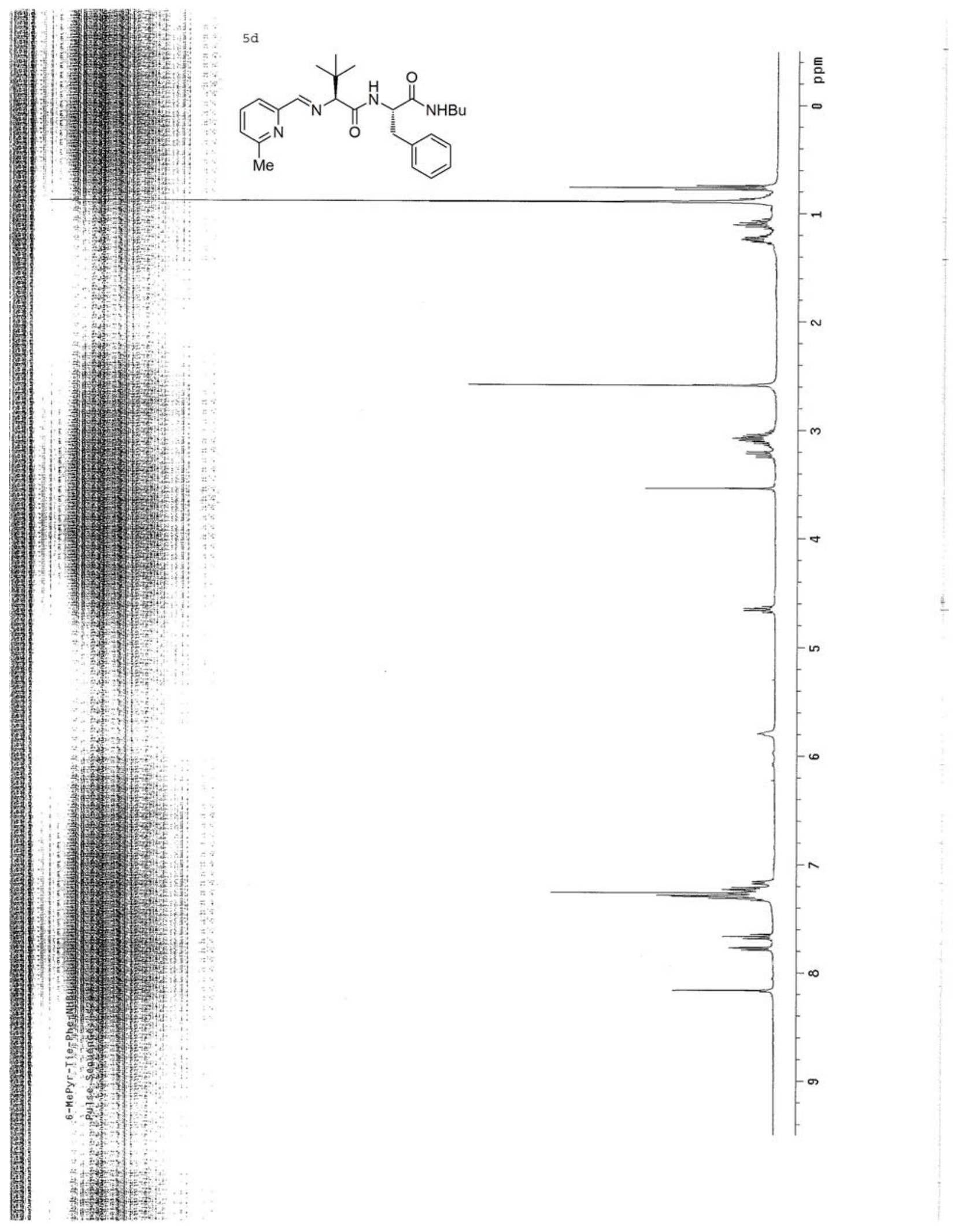




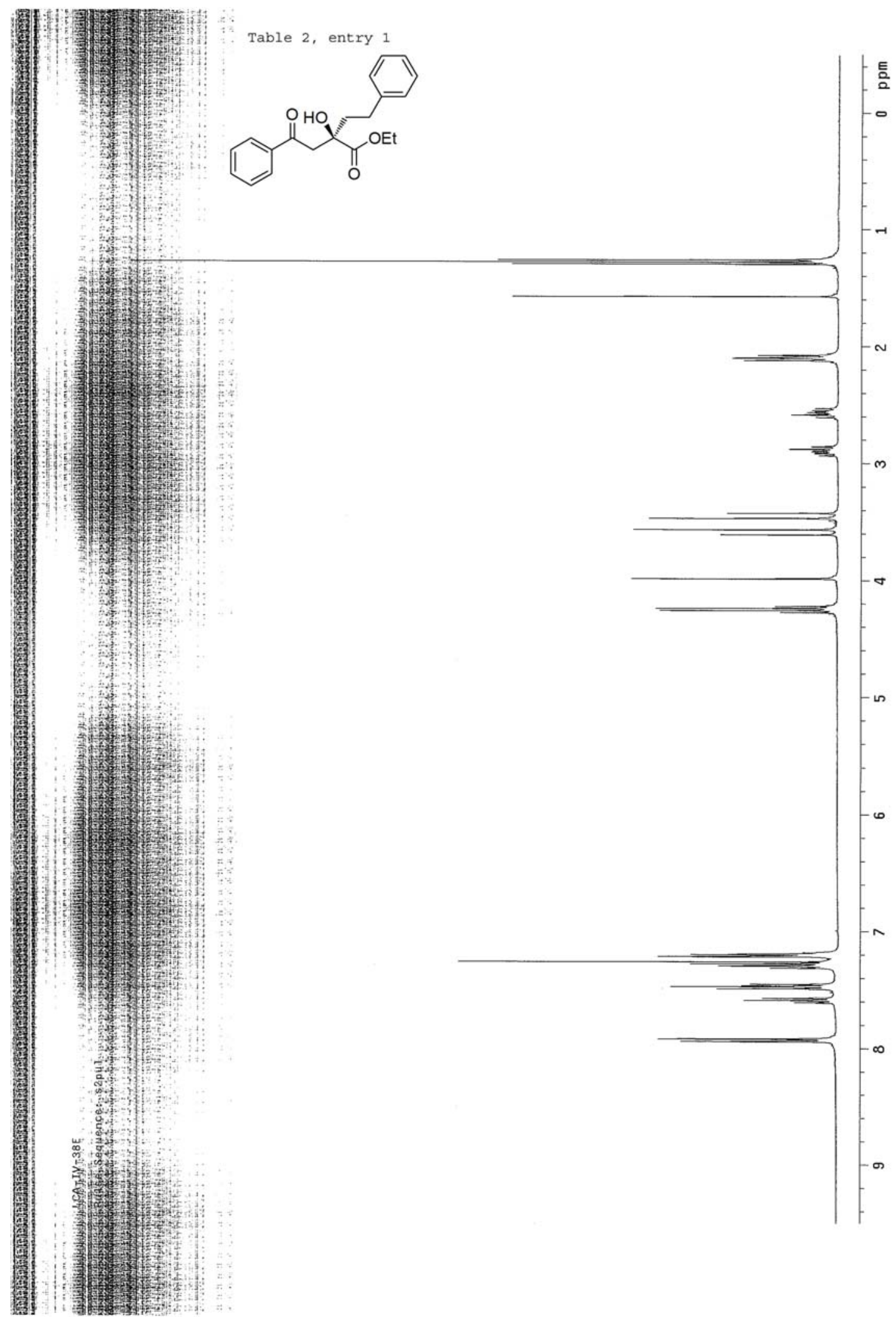




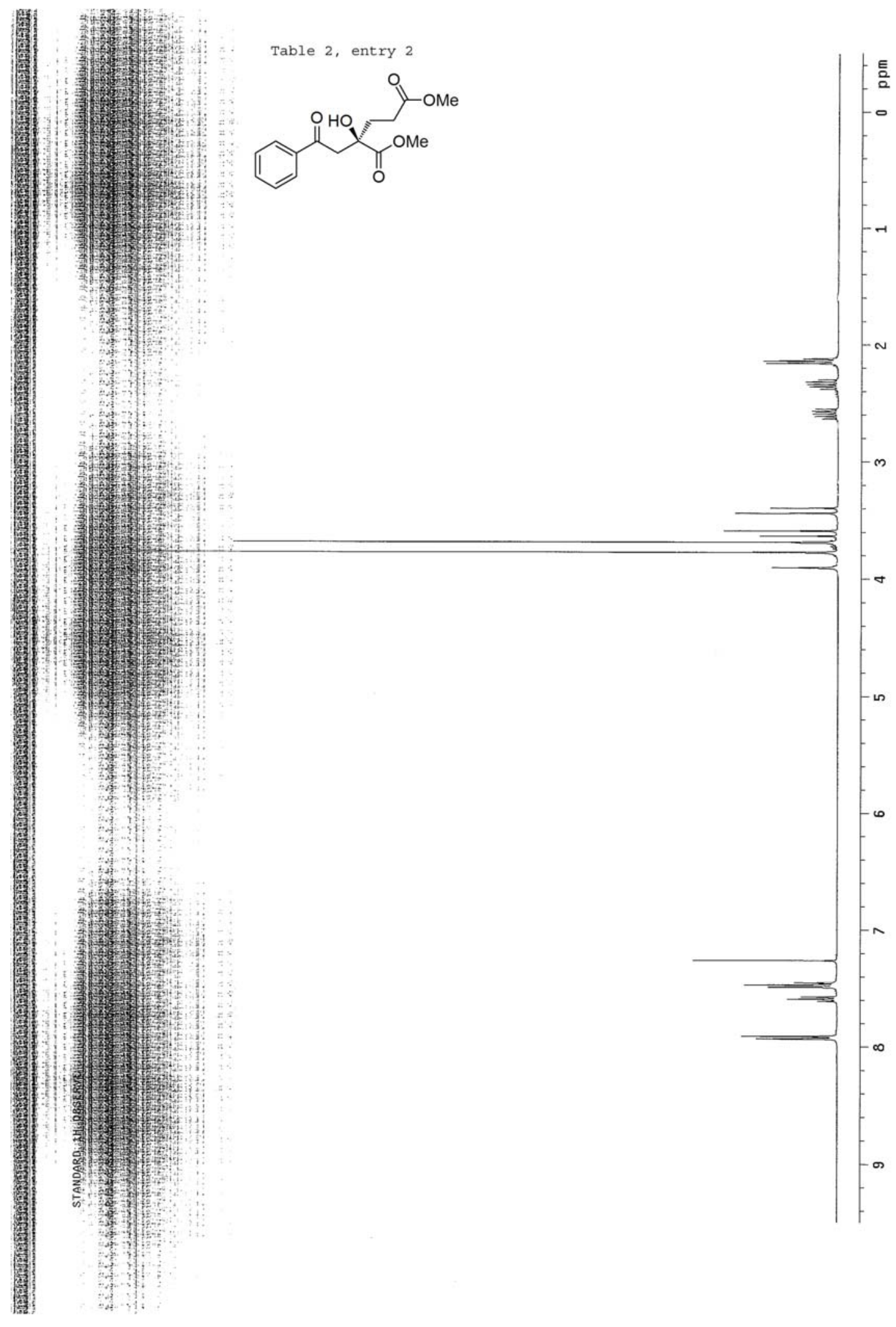




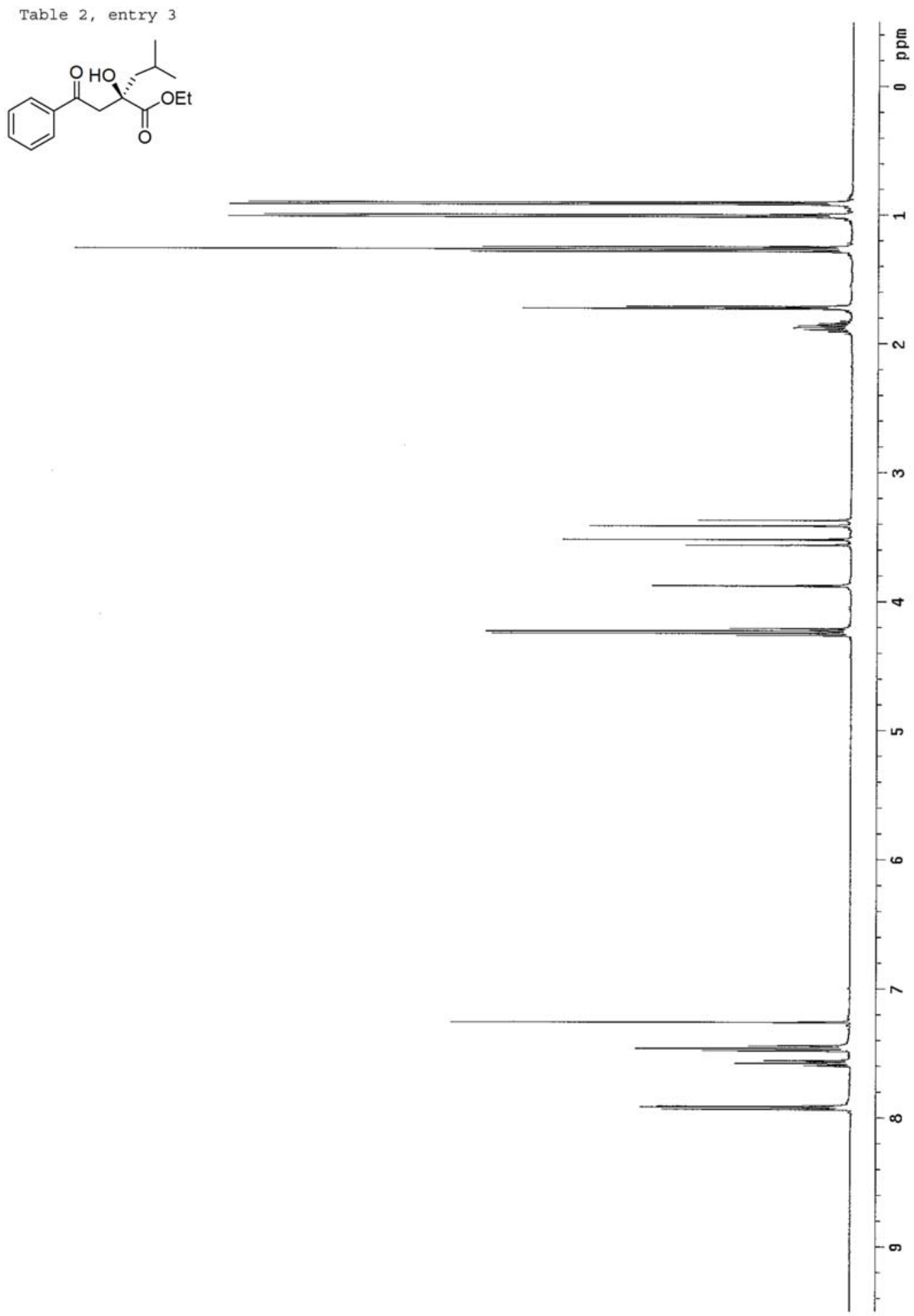




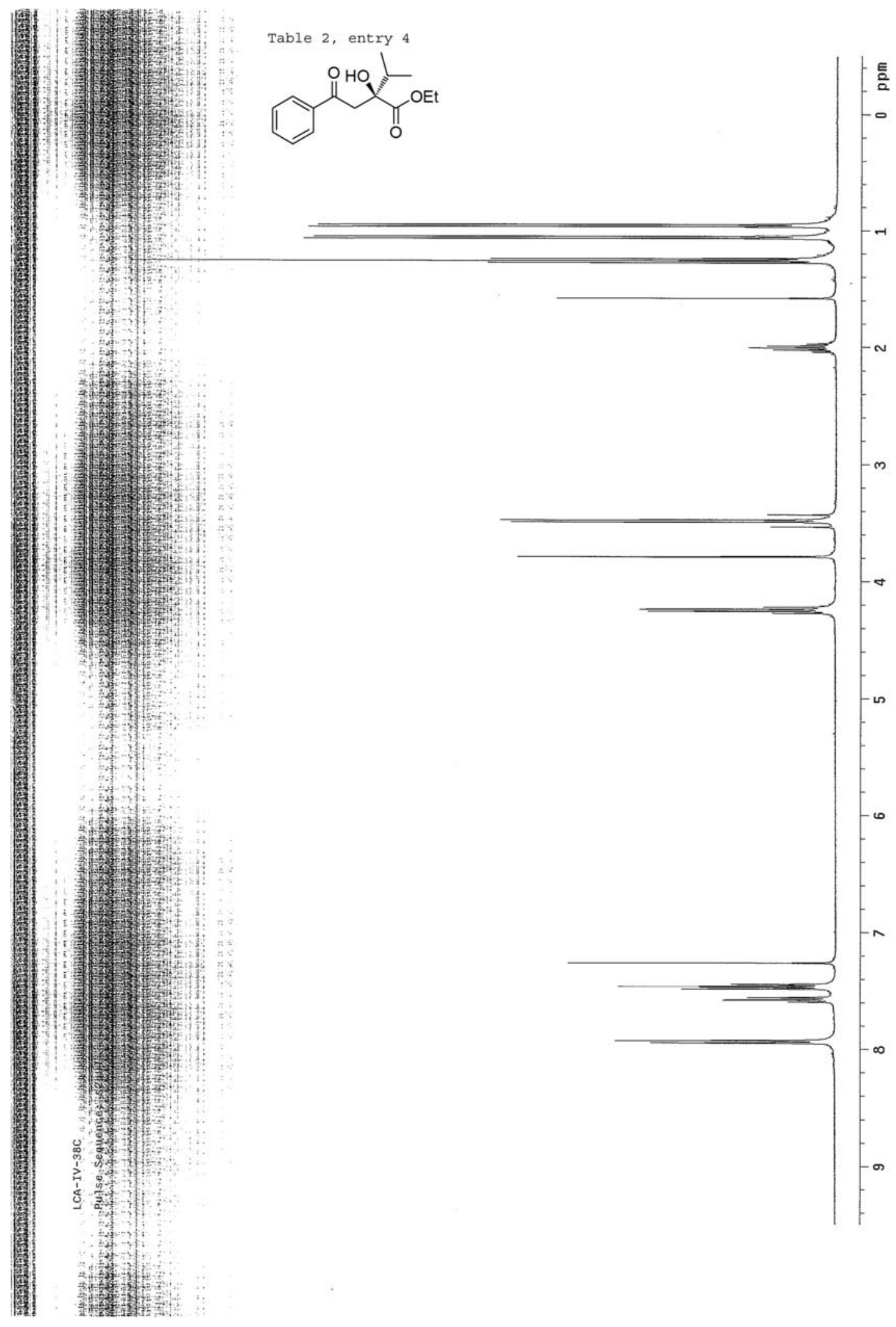


Table 2, entry 5<smiles>CCOC(=O)[C@](O)(CC(=O)C(C)(C)C)C(C)C</smiles>

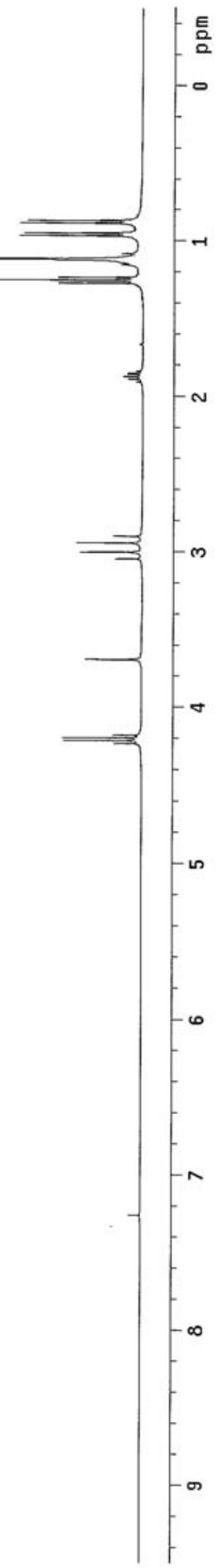


Table 2, entry 6
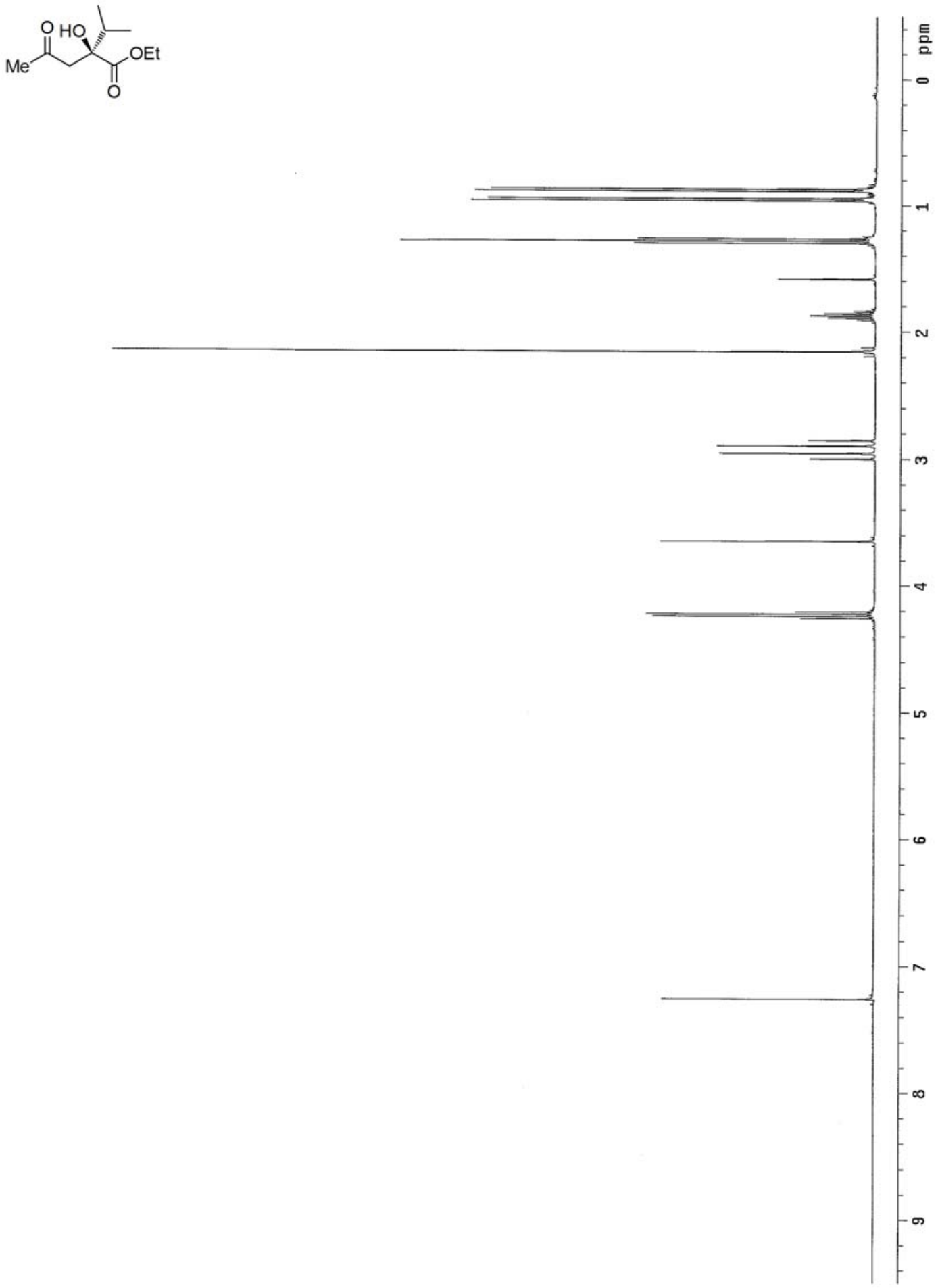

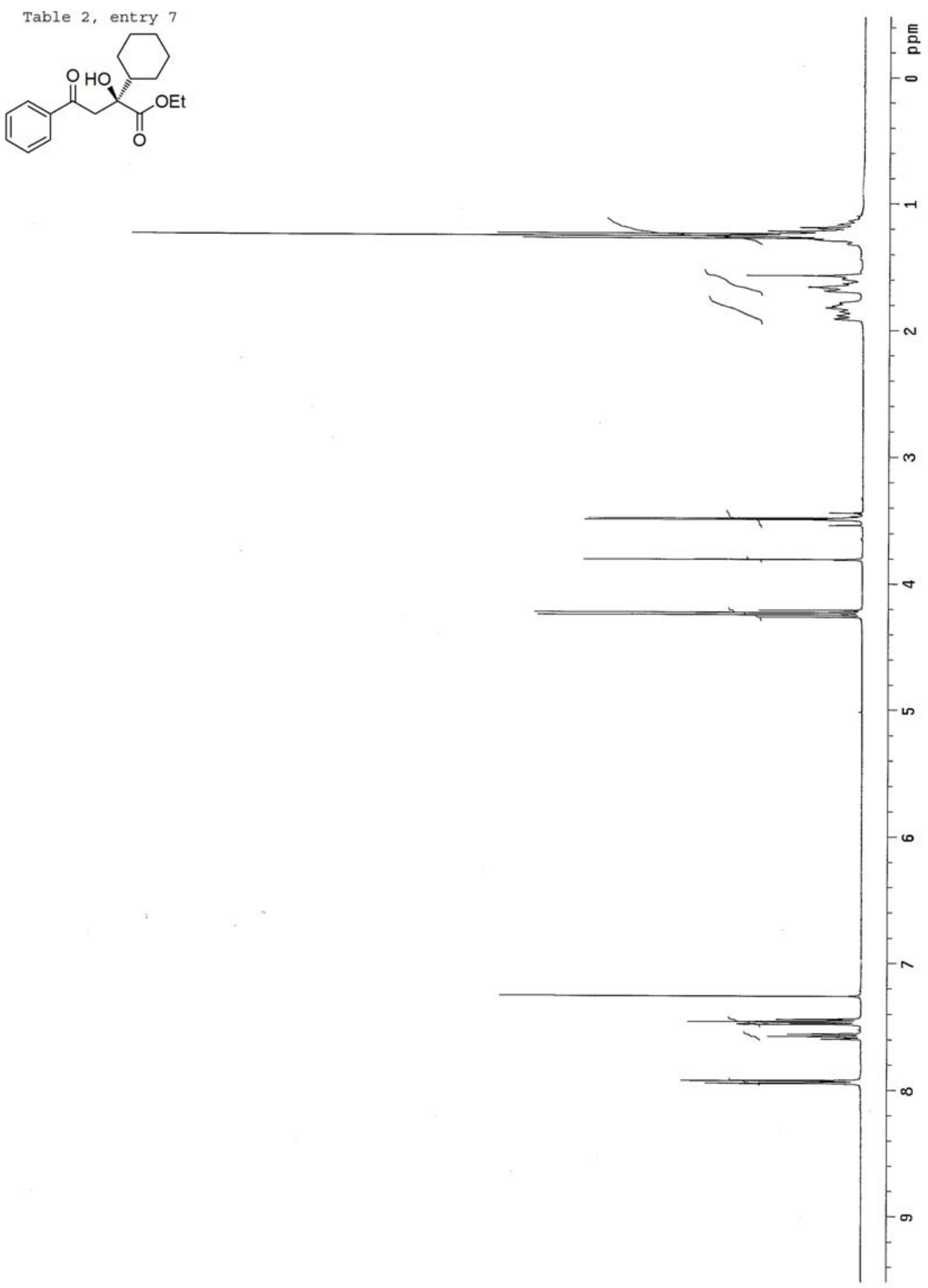
Table 2 , entry 8
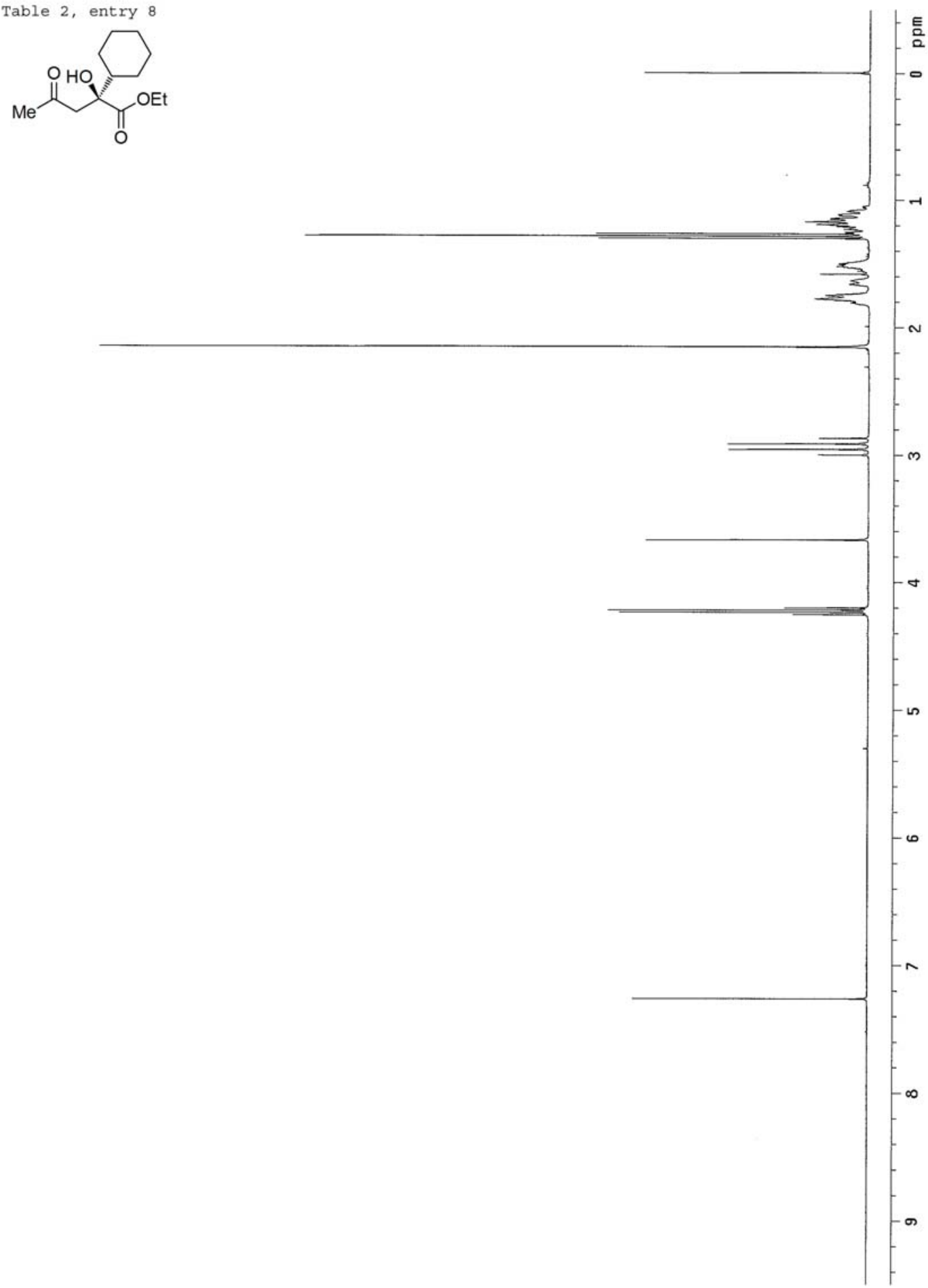


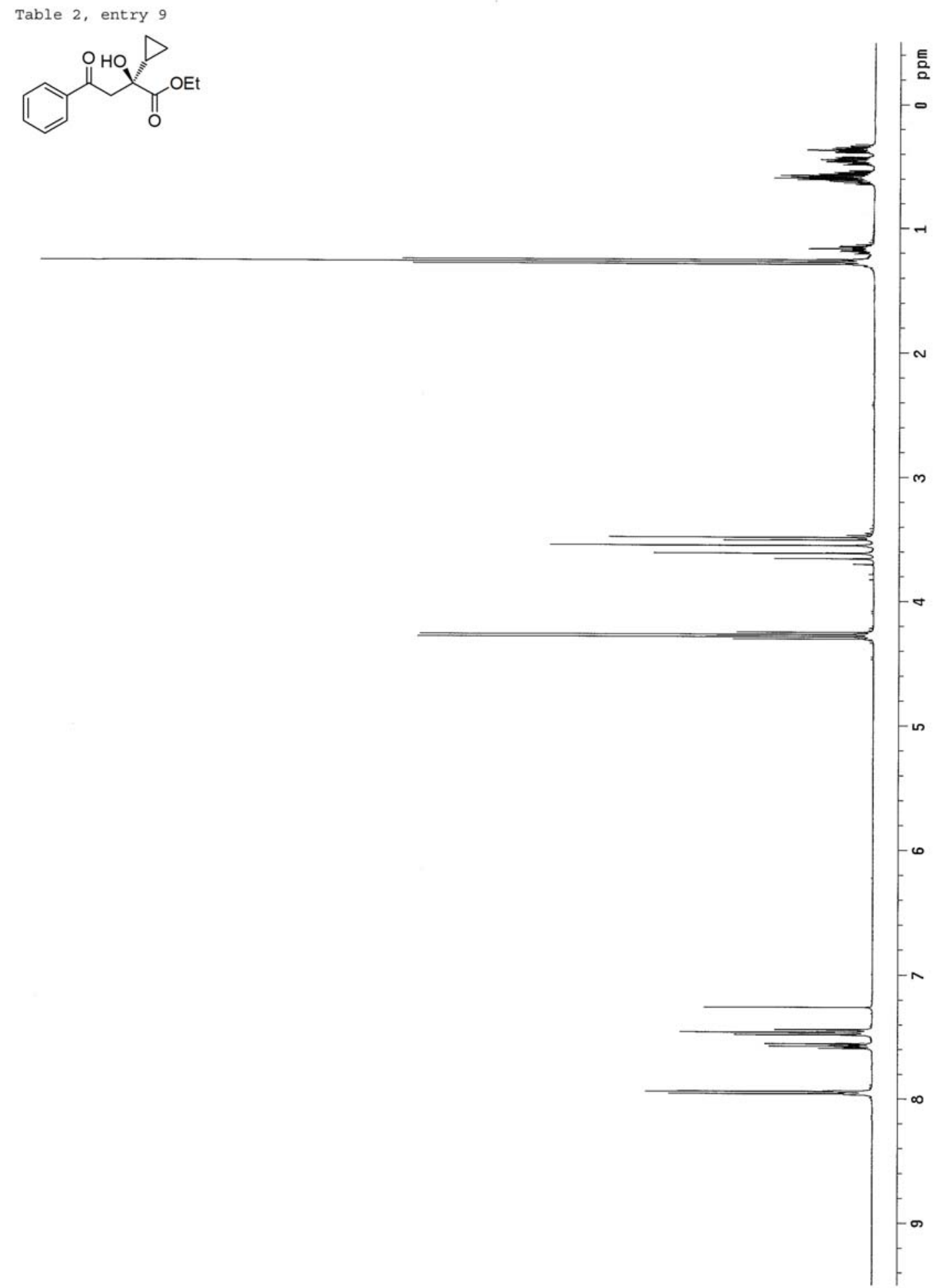




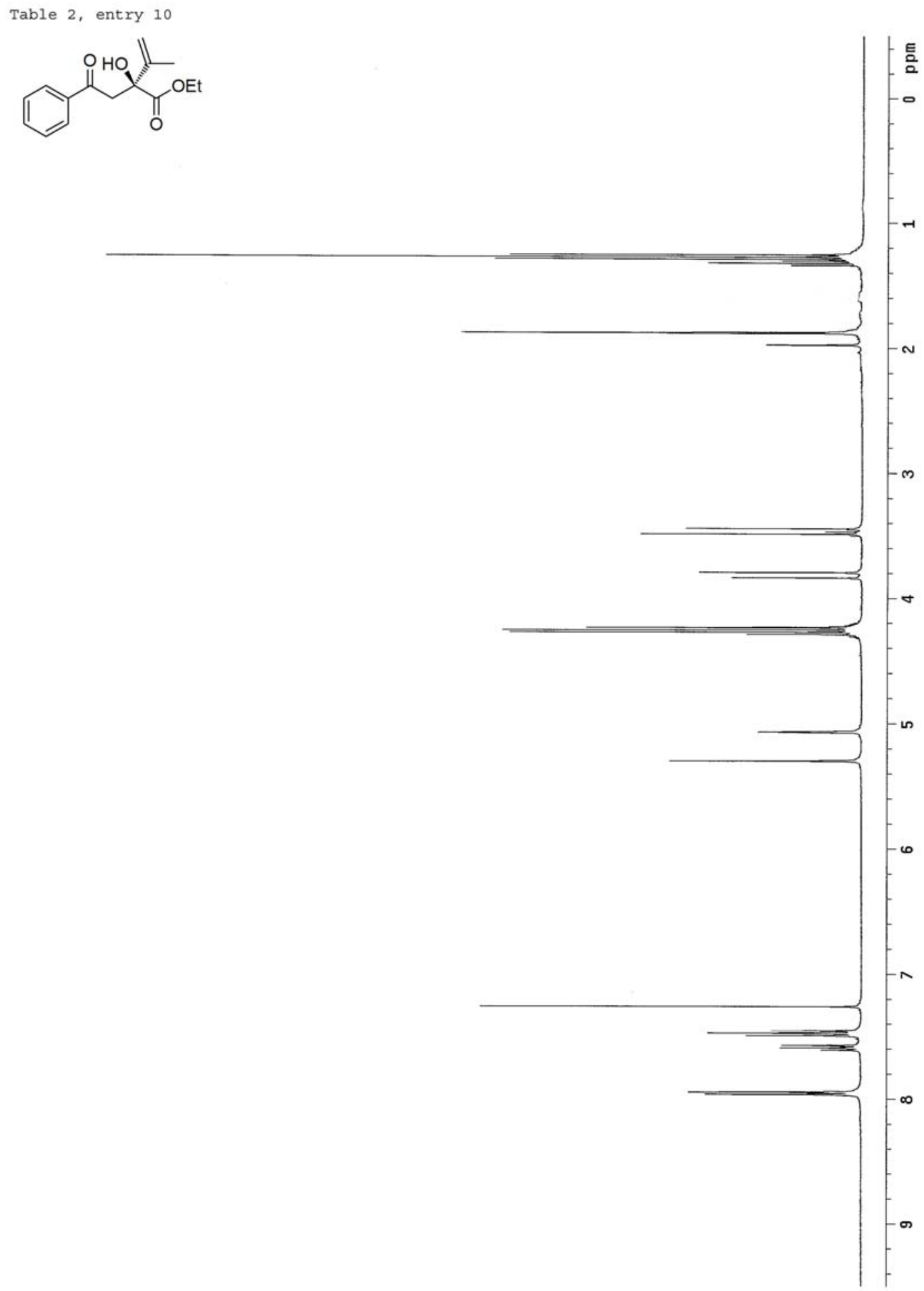



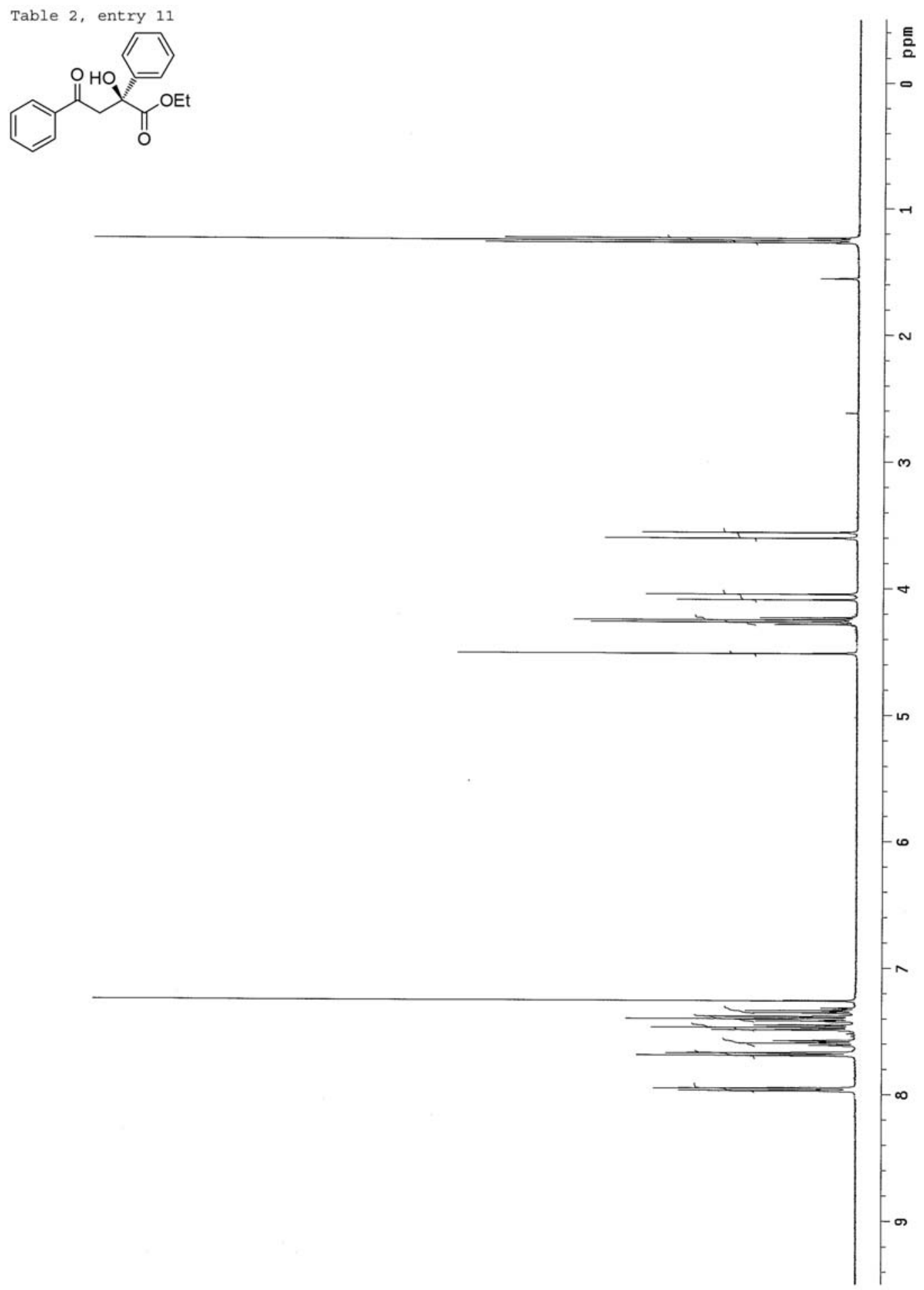
Table 2, entry 12
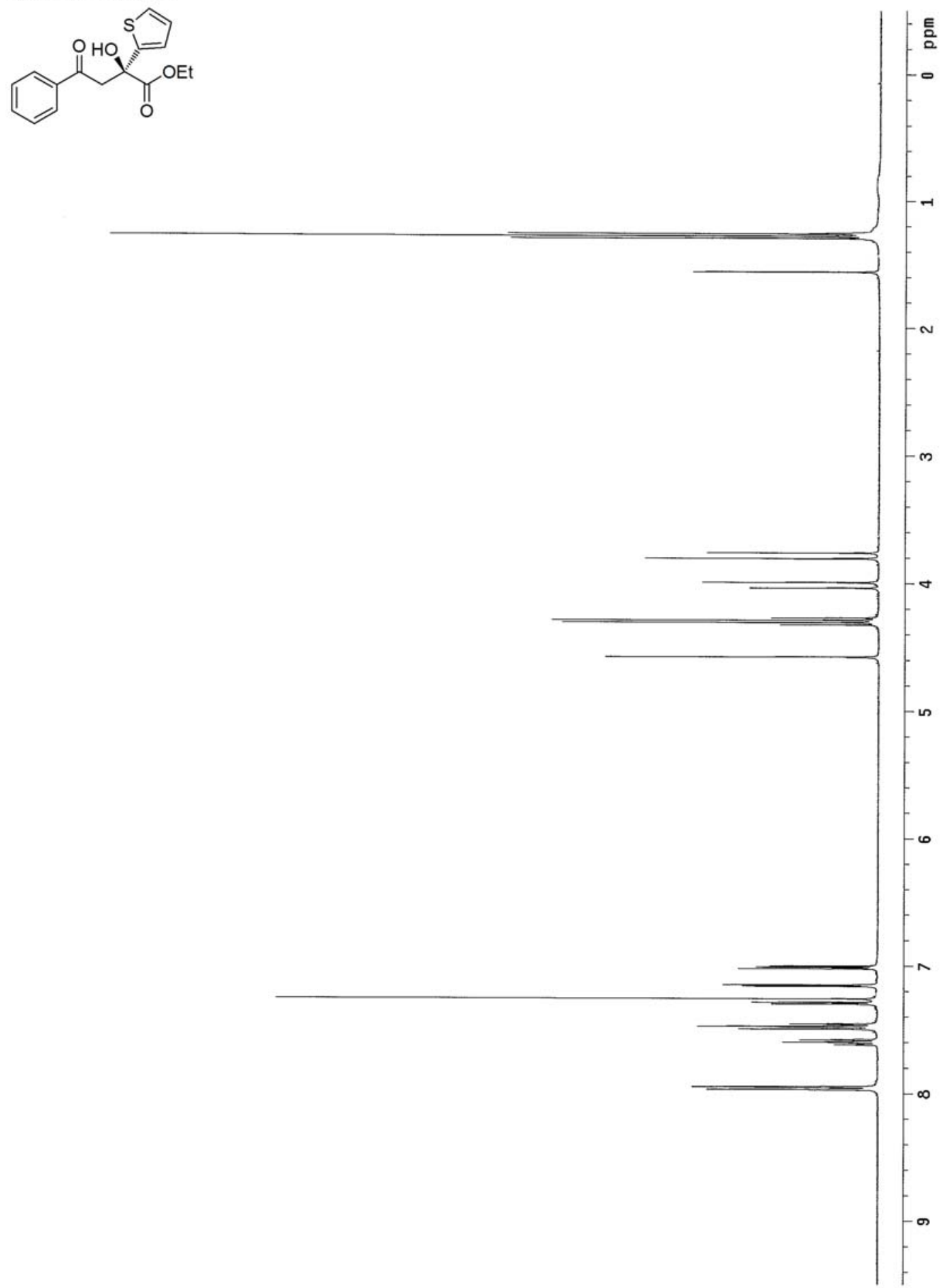

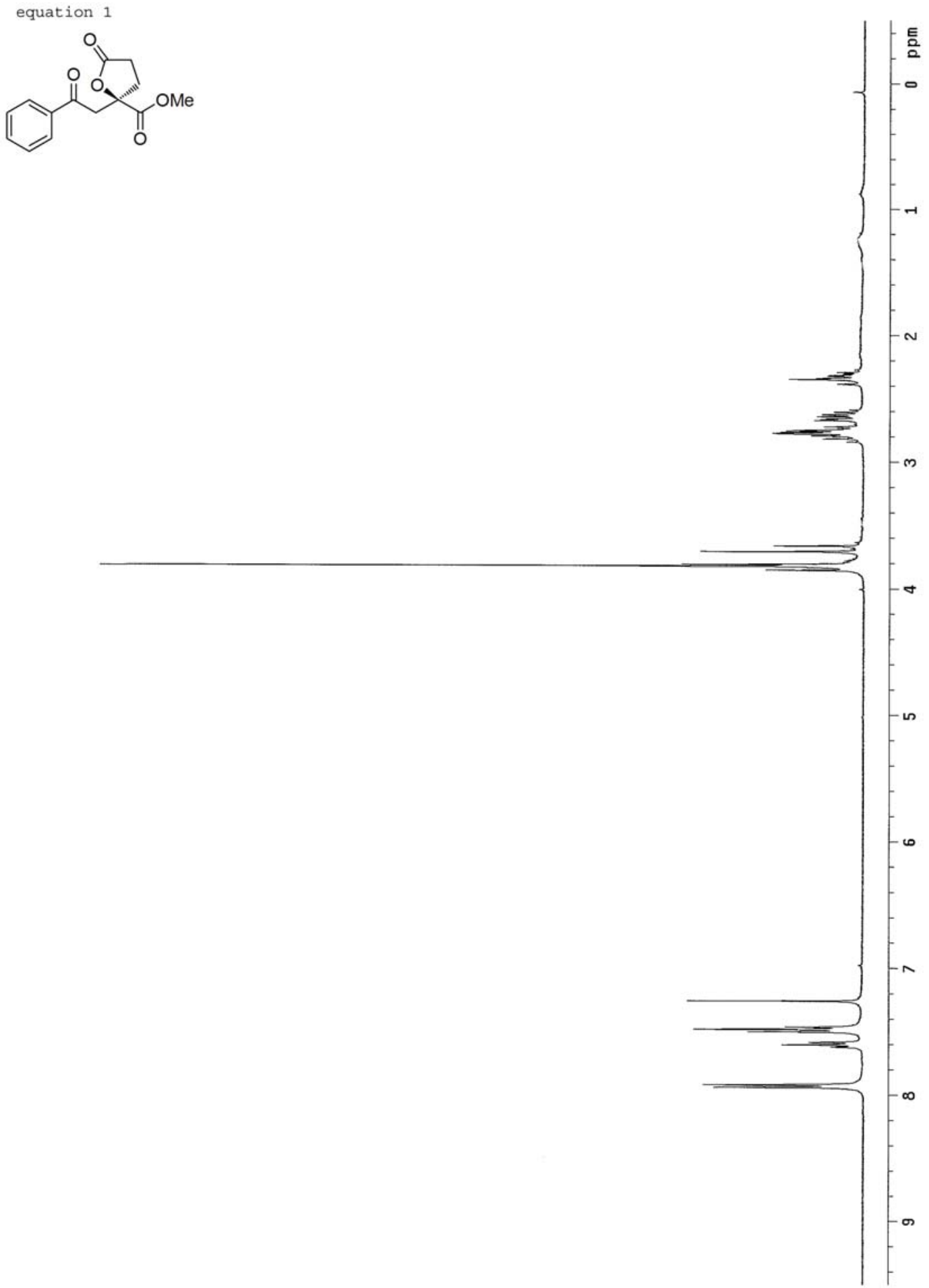


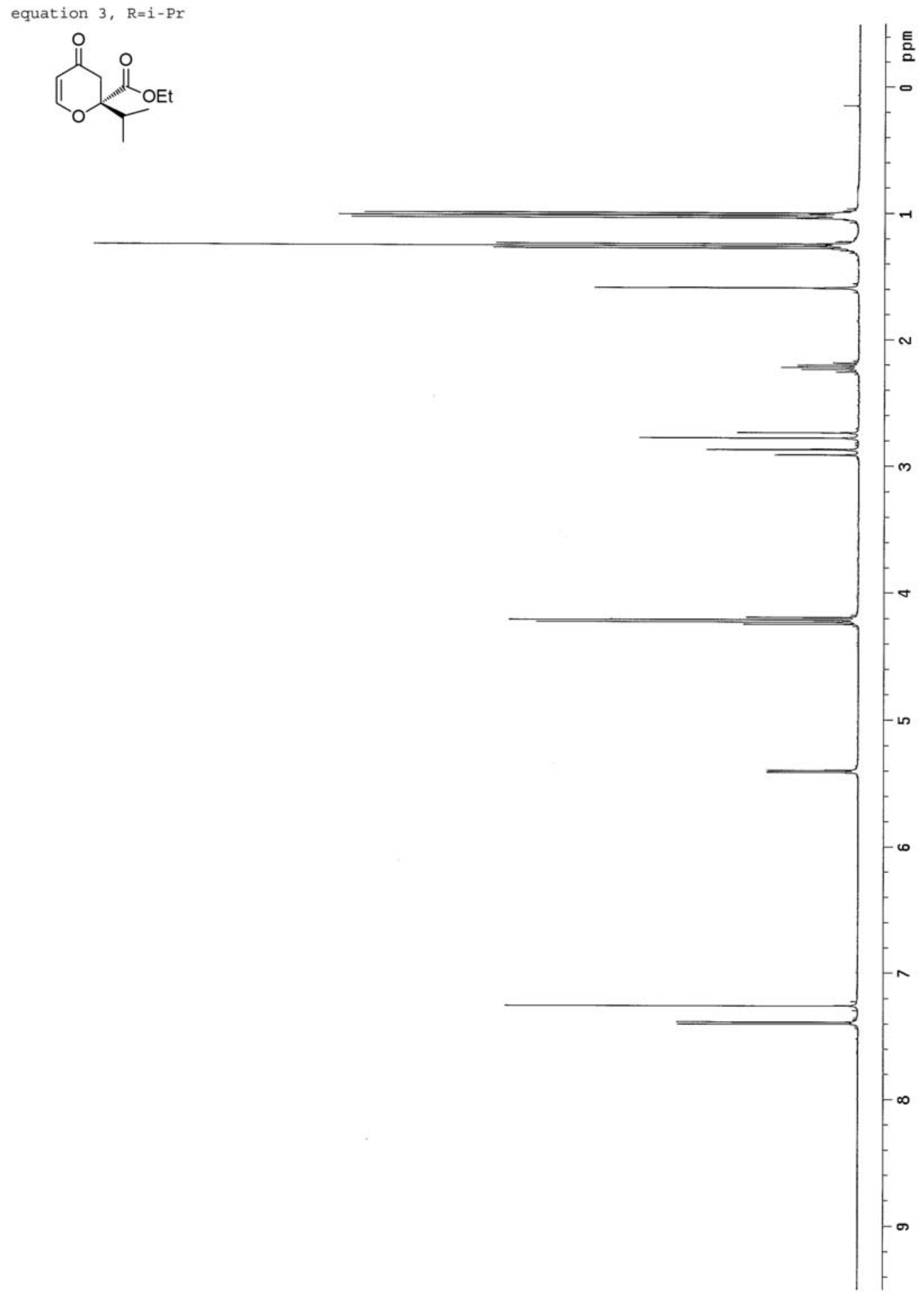




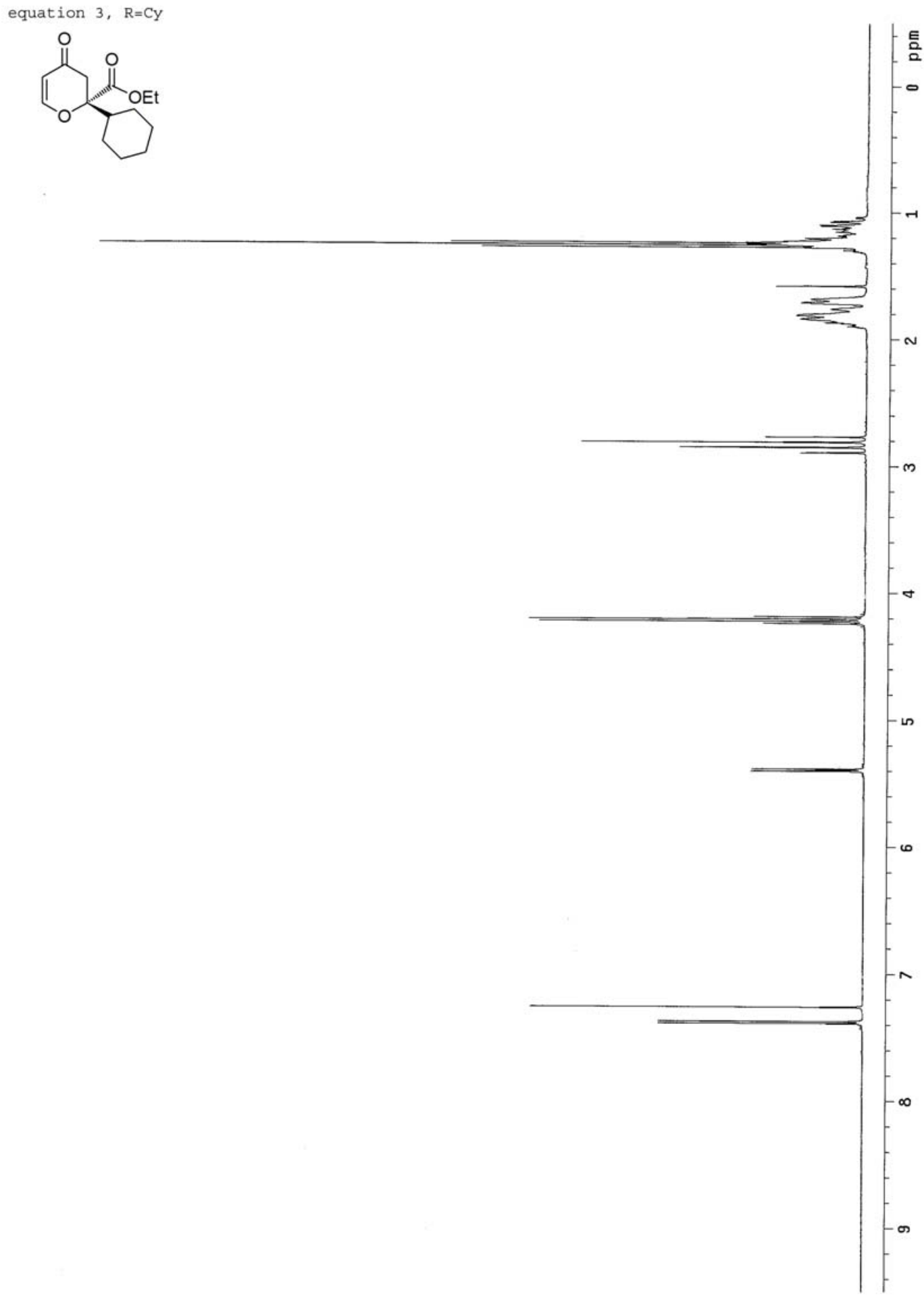



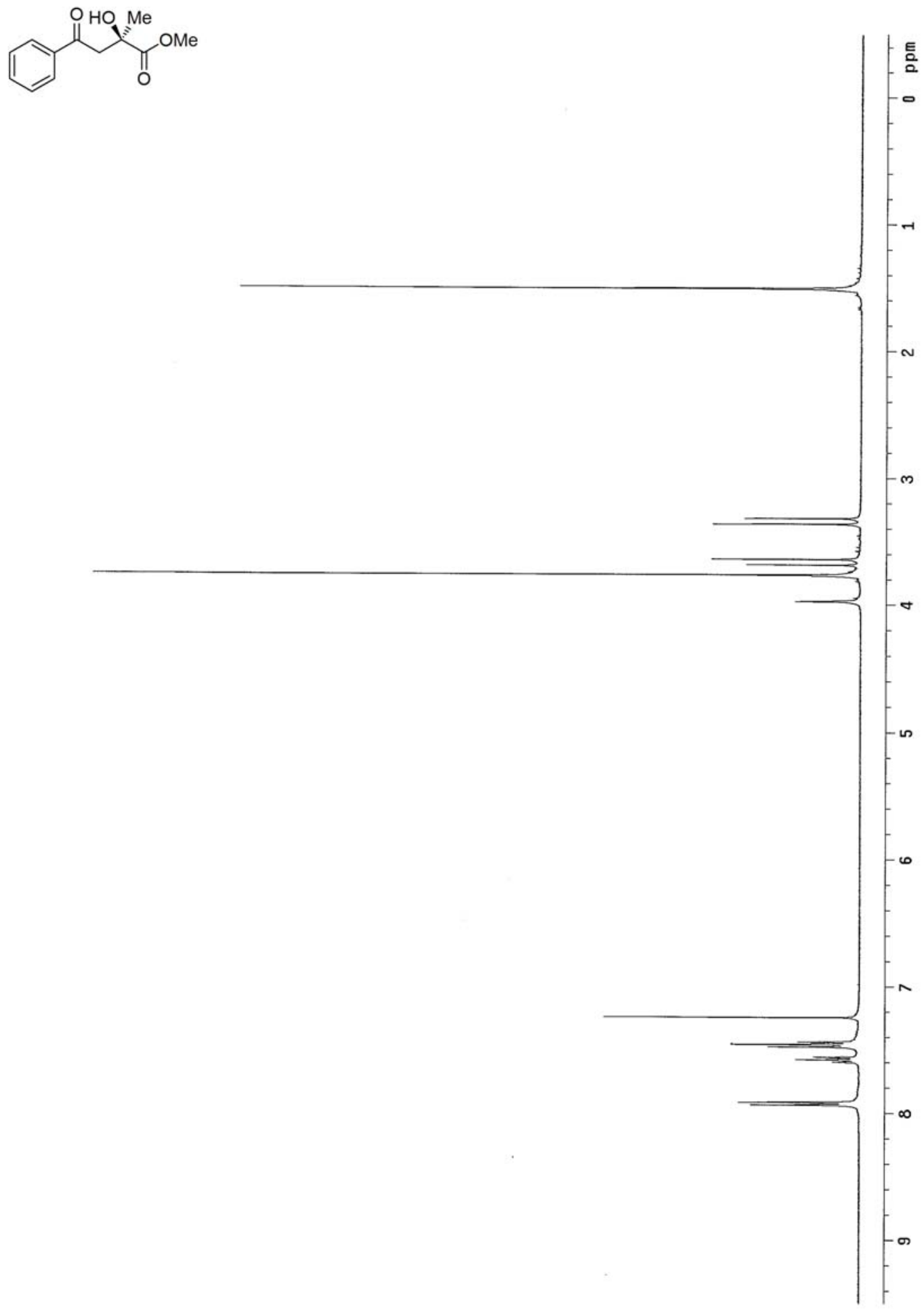